\title{
MODELLING ASYMMETRIC EXCHANGE RATE DEPENDENCE*
}

\author{
BY ANDREW J. PATTON ${ }^{1}$ \\ London School of Economics, U.K.
}

\begin{abstract}
We test for asymmetry in a model of the dependence between the Deutsche mark and the yen, in the sense that a different degree of correlation is exhibited during joint appreciations against the U.S. dollar versus during joint depreciations. We consider an extension of the theory of copulas to allow for conditioning variables, and employ it to construct flexible models of the conditional dependence structure of these exchange rates. We find evidence that the mark-dollar and yen-dollar exchange rates are more correlated when they are depreciating against the dollar than when they are appreciating.
\end{abstract}

\section{INTRODUCTION}

Evidence that the univariate distributions of many common economic variables are nonnormal has been widely reported, as far back as Mills (1927). Common examples of deviations from normality include excess kurtosis (or fat tails) and skewness in univariate distributions. Recent studies of equity returns have also reported deviations from multivariate normality, in the form of asymmetric dependence. One example of asymmetric dependence is where two returns exhibit greater correlation during market downturns than market upturns, as reported in Erb et al. (1994), Longin and Solnik (2001), and Ang and Chen (2002). Various explanations for the presence of asymmetric dependence between equity returns have been proffered. For example, Ribeiro and Veronesi (2002) suggest correlations between international stock markets increase during market downturns as a consequence of investors having greater uncertainty about the state of the economy.

Much less attention has been paid to the possibility of asymmetric dependence between exchange rates. Asymmetric responses of central banks to exchange rate movements is a possible cause of asymmetric dependence. For example, a desire

\footnotetext{
* Manuscript received June 2003; revised January 2005.

1 This article is based on Chapter I of my Ph.D. dissertation, Patton (2002), and was previously circulated under the title "Modelling Time-Varying Exchange Rate Dependence Using the Conditional Copula." I would like to thank Graham Elliott, Rob Engle, Raffaella Giacomini, Tony Hall, Joshua Rosenberg, Kevin Sheppard, Allan Timmermann, four anonymous referees, and seminar participants at the Econometric Society meetings in Maryland, Board of Governors of the Federal Reserve, Chicago Graduate School of Business, Commonwealth Scientific and Industrial Research Organisation, London School of Economics, Michigan State, Monash, Oxford, Pennsylvania, Princeton, Purdue, Texas A\&M, UCSD, UC-Riverside, University of Technology Sydney, and Yale for their comments and suggestions. Financial support from the UCSD Project in Econometric Analysis Fellowship is gratefully acknowledged. Please address correspondence to: A. J. Patton, Financial Markets Group, London School of Economics, Houghton Street, London WC2A 2AE, U.K. E-mail: a.patton@lse.ac.uk.
} 
to maintain the competitiveness of Japanese exports to the United States. with German exports to the United States. would lead the Bank of Japan to intervene to ensure a matching depreciation of the yen against the dollar whenever the Deutsche mark (DM) depreciated against the U.S. dollar. Such a scenario was considered by Takagi (1999). On the other hand, a preference for price stability could lead the Bank of Japan to intervene to ensure a matching appreciation of the yen against the dollar whenever the DM appreciated against the U.S. dollar. An imbalance in these two objectives could cause asymmetric dependence between these exchange rates. If the competitiveness preference dominates the price stability preference, we would expect the DM and yen to be more dependent during depreciations against the dollar than during appreciations. An alternative cause could come from portfolio rebalancing: When the dollar strengthens there is often a shift of funds from other currencies into the dollar, whereas when the dollar weakens much of these funds shift into the DM or euro instead of the yen, as the former was/is the second most important currency. ${ }^{2}$ Such rebalancing behavior would also lead to greater dependence during depreciations of the DM and yen against the dollar than during appreciations.

To investigate whether the dependence structure of these exchange rates is asymmetric, we make use of a theorem due to Sklar (1959), which shows that any $n$-dimensional joint distribution function may be decomposed into its $n$ marginal distributions, and a copula, which completely describes the dependence between the $n$ variables. ${ }^{3}$ The copula is a more informative measure of dependence between two (or more) variables than linear correlation, as when the joint distribution of the variables of interest is nonelliptical the usual correlation coefficient is no longer sufficient to describe the dependence structure.

By using an extension of Sklar's theorem, we are able to exploit the success we have had in the modeling of univariate densities by first specifying models for the marginal distributions of a multivariate distribution of interest, and then specifying a copula. For example, consider the modeling of the joint distribution of two exchange rates: The Student's $t$ distribution has been found to provide a reasonable fit to the conditional univariate distribution of daily exchange rate returns; see Bollerslev (1987) among others. A natural starting point in the modeling of the joint distribution of two exchange rates might then be a bivariate $t$ distribution. However, the standard bivariate Student's $t$ distribution has the restrictive property that both marginal distributions have the same degrees of freedom parameter. Studies such as Bollerslev (1987) have shown that different exchange rates have different degrees of freedom parameters, and our empirical results confirm that this is true for the Deutsche mark-U.S. dollar and yen-U.S. dollar exchange rates: The restriction that both exchange rate returns have the same degrees of freedom

\footnotetext{
${ }^{2}$ I thank a referee for providing these further suggestions on possible sources of asymmetric exchange rate dependence.

3 The word copula comes from Latin for a "link" or "bond," and was coined by Sklar (1959), who first proved the theorem that a collection of marginal distributions can be "coupled" together via a copula to form a multivariate distribution. It has been given various names, such as dependence function (Galambos, 1978, and Deheuvels, 1978), uniform representation (Kimeldorf and Sampson, 1975, and Hutchinson and Lai, 1990), or standard form (Cook and Johnson, 1981).
} 
parameter is rejected by the data. Note also that this is possibly the most ideal situation: where both assets turn out to have univariate distributions from the same family, the Student's $t$, and very similar degrees of freedom (6.2 for the mark and 4.3 for the yen). We could imagine situations where the two variables of interest have quite different marginal distributions, where no obvious choice for the bivariate density exists. Further, the bivariate Student's $t$ distribution imposes a symmetric dependence structure, ruling out the possibility that the exchange rates may be more or less dependent during appreciations than during depreciations. Decomposing the multivariate distribution into the marginal distributions and the copula allows for the construction of better models of the individual variables than would be possible if we constrained ourselves to look only at existing multivariate distributions.

A useful parametric alternative to copula-based multivariate models is a multivariate regime switching model; see Ang and Bekaert (2002) for example. These authors show that a mixture of two multivariate normal distributions can match the asymmetric equity-return dependence found in Longin and Solnik (2001), and thus may also be useful for studying asymmetric exchange rate dependence. A detailed comparison of flexible copula-based models and flexible multivariate regime switching models for exchange rates and/or equity returns would be an interesting study, but we leave it for future research.

An alternative to parametric specifications of the multivariate distribution would, of course, be a nonparametric estimate, as in Fermanian and Scaillet (2003) for example, which can accommodate all possible distributional forms. One common drawback with nonparametric approaches is the lack of precision that occurs when the dimension of the distribution of interest is moderately large (say over four), or when we consider multivariate distributions conditioned on a state vector (as is the case in this article). The trade-off for this lack of precision is the fact that a parametric specification may be misspecified. It is for this reason that we devote a great deal of attention to tests of goodness-of-fit of the proposed specifications.

This article makes two contributions. Our first contribution is to consider how the theory of (unconditional) copulas may be extended to the conditional case, thus allowing us to use copula theory in the analysis of time-varying conditional dependence. Time variation in the conditional first and second moments of economic time series has been widely reported, and so allowing for time variation in the conditional dependence between economic time series seems natural. The second and main contribution of the article is to show how we may use conditional copulas for multivariate density modeling. We examine daily Deutsche mark-U.S. dollar (DM-USD) and Japanese yen-U.S. dollar (Yen-USD) exchange rates over the period January 1991 to December 2001, and propose a new copula that allows for asymmetric dependence and includes symmetric dependence as a special case. We find significant evidence that the dependence structure between the DM-USD and Yen-USD exchange rates was asymmetric, consistent with the asymmetric central bank behavior story presented above. We also find very strong evidence of a structural break in the conditional copula following the introduction of the euro in January 1999: The level of dependence drops substantially, the dynamics of conditional dependence change, and the dependence structure 
goes from significantly asymmetric in one direction to weakly asymmetric in the opposite direction.

The modeling of the entire conditional joint distribution of these exchange rates has a number of attractive features: Given the conditional joint distribution, we can, of course, obtain conditional means, variances, and correlation, as well as the time paths of any other dependence measure of interest, such as rank correlation or tail dependence. ${ }^{4}$ Further, there are economic situations where the entire conditional joint density is required, such as the pricing of financial options with multiple underlying assets (see Rosenberg, 2003) or in the calculation of portfolio Value-at-Risk (VaR) (see Hull and White, 1998) or in a forecast situation where the loss function of the forecast's end user is unknown.

Despite the fact that copulas were introduced as a means of isolating the dependence structure of a multivariate distribution over 40 years ago, it is only recently that they attracted the attention of economists. In the last few years, numerous papers have appeared, using copulas in such applications as multivariate option pricing, asset allocation, models of default risk, integrated risk management, selectivity bias, nonlinear autoregressive dependence, and contagion. ${ }^{5}$ To our knowledge, this article is one of the first to consider copulas for time-varying conditional distributions, emphasize the importance of formal goodness-of-fit testing for copulas and marginal distributions, and to employ statistical tests comparing the goodness-of-fit of competing nonnested copulas.

The structure of the remainder of this article is as follows. In Section 2, we present the theory of the conditional copula. In Section 3, we apply the theory of conditional copulas to a study of the dependence structure of the Deutsche markU.S. dollar and yen-U.S. dollar exchange rates. In that section, we discuss the construction and evaluation of time-varying conditional copula models. We summarize our results in Section 4. Details on the goodness-of-fit tests are presented in the Appendix.

\section{THE CONDITIONAL COPULA}

In this section we review the theory of copulas and discuss the extension to handle conditioning variables. Though in this article we focus on bivariate distributions, it should be noted that the theory of copulas is applicable to the more general multivariate case. We must first define the notation: The variables of interest are $X$ and $Y$ and the conditioning variable is $W$, which may be a vector. Let the joint distribution of $(X, Y, W)$ be $F_{X Y W}$, denote the conditional distribution of $(X, Y)$ given $W$, as $F_{X Y \mid W}$, and let the conditional marginal distributions of $X \mid W$ and $Y \mid W$ be denoted $F_{X \mid W}$ and $F_{Y \mid W}$, respectively. Recall that

\footnotetext{
${ }^{4}$ This measure will be discussed in more detail in Section 3. Dependence during extreme events has been the subject of much analysis in the financial contagion literature; see Hartmann et al. (2004) among others.

${ }^{5}$ See Frees et al. (1996), Bouyé et al. (2000a, 2000b), Cherubini and Luciano (2001, 2002), Costinot et al. (2000), Li (2000), Fermanian and Scaillet (2003), Embrechts et al. (2001), Granger et al. (forthcoming), Frey and McNeil (2001), Rockinger and Jondeau (2001), Sancetta and Satchell (2001), Smith (2003), Rodriguez (2003), Rosenberg (2003), Cherubini et al. (2004), Patton (2004a), Rosenberg and Schuermann (2004), and Chen and Fan (2006).
} 
$F_{X \mid W}(x \mid w)=F_{X Y \mid W}(x, \infty \mid w)$ and $F_{Y \mid W}(y \mid w)=F_{X Y \mid W}(\infty, y \mid w)$. We will assume in this article that the distribution function $F_{X Y W}$ is sufficiently smooth for all required derivatives to exist, and that $F_{X \mid W}, F_{Y \mid W}$, and $F_{X Y \mid W}$ are continuous. The latter assumptions are not necessary, but making them simplifies the presentation. Throughout this article, we will denote the distribution (or c.d.f.) of a random variable using an uppercase letter, and the corresponding density (or p.d.f.) using the lowercase letter. We will denote the extended real line as $\bar{R} \equiv \mathbb{R} \cup\{ \pm \infty\}$. We adopt the usual convention of denoting random variables in upper case, $X_{t}$, and realizations of random variables in lower case, $x_{t}$.

A thorough review of (unconditional) copulas may be found in Nelsen (1999) and Joe (1997). Briefly, copula theory enables us to decompose a joint distribution into its marginal distributions and its dependence function, or copula:

$$
\begin{aligned}
F_{X Y}(x, y) & =C\left(F_{X}(x), F_{Y}(y)\right), \quad \text { or } \\
f_{x y}(x, y) & =f_{x}(x) \cdot f_{y}(y) \cdot c\left(F_{X}(x), F_{Y}(y)\right)
\end{aligned}
$$

where Equation (1) above decomposes a bivariate $c d f$, and Equation (2) decomposes a bivariate density. To provide some idea as to the flexibility that copula theory gives us, we now consider various bivariate distributions, all with standard normal marginal distributions and all implying a linear correlation coefficient, $\rho$, of 0.5 . The contour plots of these distributions are presented in Figure 1. In the upper left corner of this figure is the standard bivariate normal distribution with $\rho=0.5$. The other elements of this figure show the dependence structures implied by other copulas, with each copula calibrated so as to also yield $\rho=0.5$. It is quite clear that knowing the marginal distributions and linear correlation is not sufficient to describe a joint distribution: Clayton's copula, for example, has contours that are quite peaked in the negative quadrant, implying greater dependence for joint negative events than for joint positive events. Gumbel's copula implies the opposite. The functional form of the symmetrized Joe-Clayton will be given in Section 3; the remaining copula functional forms may be found in Joe (1997) or Patton (2004).

Now let us focus on the modifications required for the extension to conditional distributions. Assume below that the dimension of the conditioning variable, $W$, is 1 . Then the conditional bivariate distribution of $(X, Y) \mid W$ can be derived from the unconditional joint distribution of $(X, Y, W)$ as follows:

$$
F_{X Y \mid W}(x, y \mid w)=f_{w}(w)^{-1} \cdot \frac{\partial F_{X Y W}(x, y, w)}{\partial w}, \quad \text { for } w \in \mathcal{W}
$$

where $f_{w}$ is the unconditional density of $W$, and $\mathcal{W}$ is the support of $W$. However, the conditional copula of $(X, Y) \mid W$ cannot be derived from the unconditional copula of $(X, Y, W)$; further information is required. ${ }^{6}$ One definition of the conditional copula of $(X, Y) \mid W$ is given below.

\footnotetext{
${ }^{6}$ We thank a referee for pointing out that the conditional copula can be obtained given just the unconditional copula of $(X, Y, W)$ and the marginal density of $W$.
} 

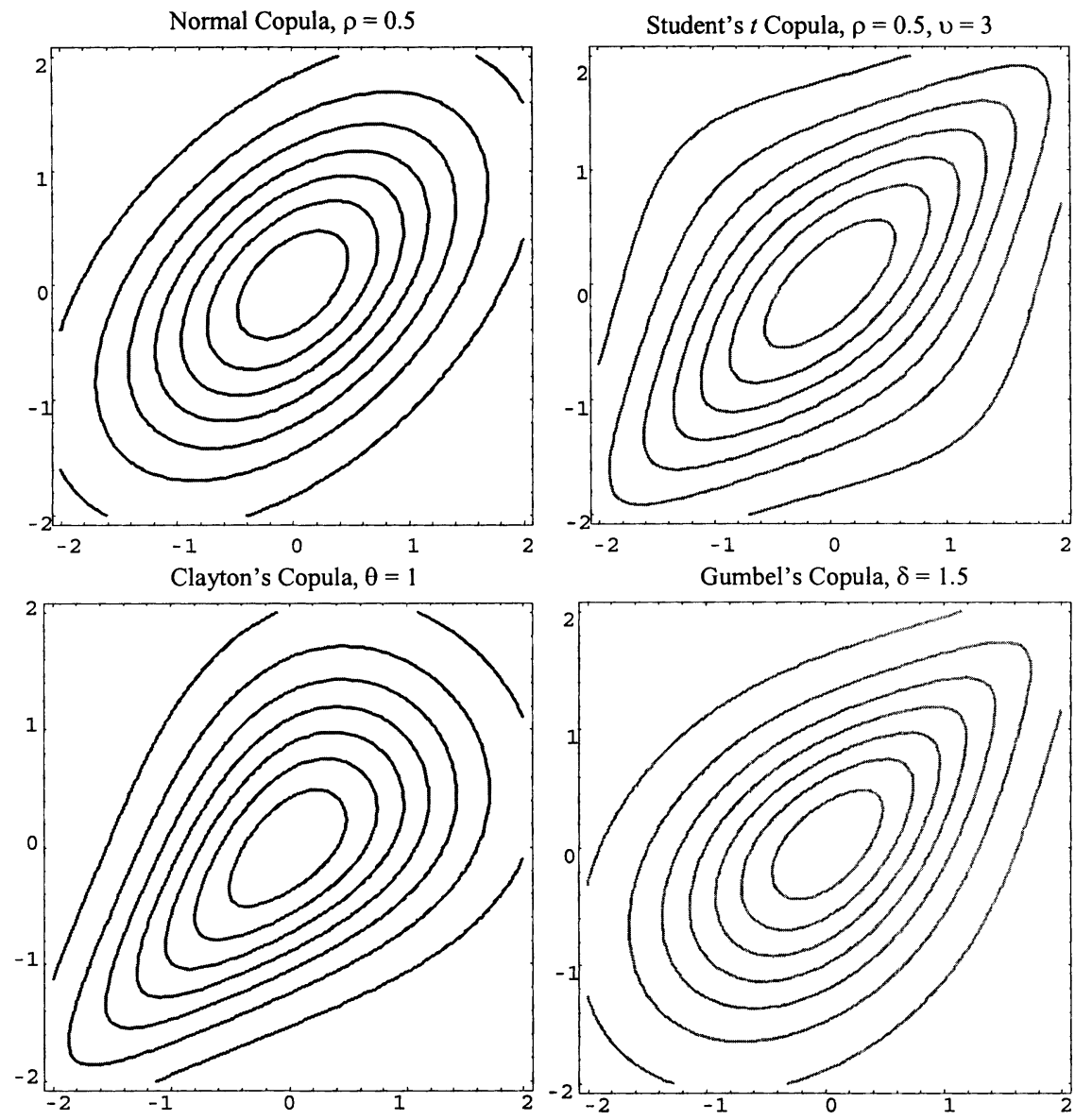

Symmetrised Joe-Clayton Copula, $\tau^{\mathrm{U}}=.45, \tau^{\mathrm{L}}=.20$

Plackett Copula, $\psi=5$
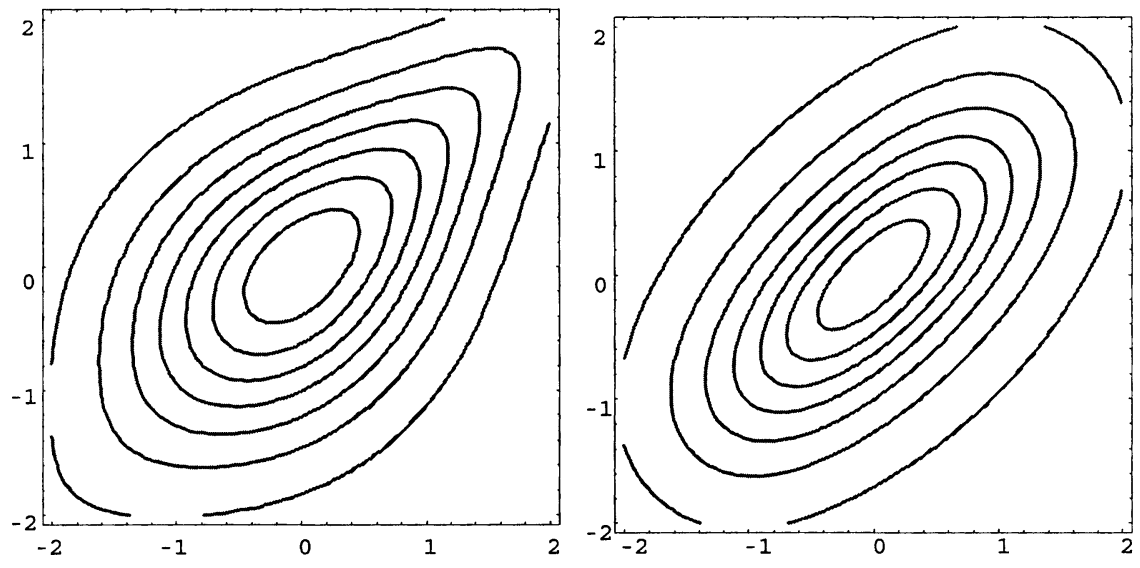

FIGURE 1

CONTOUR PLOTS OF VARIOUS DISTRIBUTIONS ALL WITH STANDARD NORMAL MARGINAL DISTRIBUTIONS AND LINEAR CORRELATION COEFFICIENTS OF 0.5 
Definition 1. The conditional copula of $(X, Y) \mid W=w$, where $X \mid W=$ $w \sim F_{X \mid W}(\cdot \mid w)$ and $Y \mid W=w \sim F_{Y \mid W}(\cdot \mid w)$, is the conditional joint distribution function of $U \equiv F_{X \mid W}(X \mid w)$ and $V \equiv F_{Y \mid W}(Y \mid w)$ given $W=w$.

The two variables $U$ and $V$ are known as the conditional "probability integral transforms" of $X$ and $Y$ given $W$. Fisher (1932) and Rosenblatt (1952) showed that these random variables have the $\operatorname{Unif}(0,1)$ distribution, regardless of the original distributions. ${ }^{7}$ It is simple to extend existing results to show that a conditional copula has the properties of an unconditional copula, for each $w \in \mathcal{W}$; see Patton (2002) for details. We now move on to an extension of Sklar's (1959); theorem for conditional distributions:

TheOREM 1. Let $F_{X \mid W}(\cdot \mid w)$ be the conditional distribution of $X \mid W=w$, $F_{Y \mid W}(\cdot \mid w)$ be the conditional distribution of $Y \mid W=w, F_{X Y \mid W}(\cdot \mid w)$ be the joint conditional distribution of $(X, Y) \mid W=w$, and $\mathcal{W}$ be the support of $W$. Assume that $F_{X \mid W}(\cdot \mid w)$ and $F_{Y \mid W}(\cdot \mid w)$ are continuous in $x$ and $y$ for all $w \in \mathcal{W}$. Then there exists a unique conditional copula $C(\cdot \mid w)$ such that

$$
\begin{aligned}
F_{X Y \mid W}(x, y \mid w)= & C\left(F_{X \mid W}(x \mid w), F_{Y \mid W}(y \mid w) \mid w\right), \\
& \forall(x, y) \in \overline{\mathbb{R}} \times \overline{\mathbb{R}} \text { and each } w \in \mathcal{W}
\end{aligned}
$$

Conversely, if we let $F_{X \mid W}(\cdot \mid w)$ be the conditional distribution of $X \mid W=w$, $F_{Y \mid W}(\cdot \mid w)$ be the conditional distribution of $Y \mid W=w$, and $\{C(\cdot \mid w)\}$ be a family of conditional copulas that is measurable in $w$, then the function $F_{X Y \mid W}(\cdot \mid w)$ defined by Equation (3) is a conditional bivariate distribution function with conditional marginal distributions $F_{X \mid W}(\cdot \mid w)$ and $F_{Y \mid W}(\cdot \mid w)$.

It is the converse of Sklar's theorem that is the most interesting for multivariate density modeling. It implies that we may link together any two univariate distributions, of any type (not necessarily from the same family), with any copula and we will have defined a valid bivariate distribution. The usefulness of this result stems from the fact that although in the economics and statistics literatures we have a vast selection of flexible parametric univariate distributions, the set of parametric multivariate distributions available is much smaller. With Sklar's theorem, the set of possible parametric bivariate distributions is increased substantially, though, of course, not all of these distributions will be useful empirically. With a corollary to Sklar's theorem, given in Nelsen (1999) for example, the set of possible parametric multivariate distributions increases even further, as we are able to extract the copula from any given multivariate distribution and use it independently of the marginal distributions of the original distribution. This corollary allows us to extract, for example, the "normal copula" from a standard bivariate normal distribution.

\footnotetext{
${ }^{7}$ The probability integral transform has also been used in the context of goodness-of-fit tests as far back as the 1930s; see Pearson (1933) for example. More recently, Diebold et al. (1998) extended the probability integral transform theory to the time series case, and proposed using it in the evaluation of density forecasts.
} 
The only complication introduced when extending Sklar's theorem to conditional distributions is that the conditioning variable(s), $W$, must be the same for both marginal distributions and the copula. This is important in the construction of conditional density models using copula theory. Failure to use the same conditioning variable for $F_{X \mid W}, F_{Y \mid W}$, and $C$ will, in general, lead to a failure of the function $F_{X Y \mid W}$ to satisfy the conditions for it to be a joint conditional distribution function. For example, say we condition $X$ on $W_{1}, Y$ on $W_{2}$, and the copula on $\left(W_{1}, W_{2}\right)$, and then specify $\tilde{F}_{X Y \mid W_{1}, W_{2}}\left(x, y \mid w_{1}, w_{2}\right)=C\left(F_{X \mid W_{1}}\left(x \mid w_{1}\right), F_{Y \mid W_{2}}\left(y \mid w_{2}\right) \mid w_{1}, w_{2}\right)$. Then $\tilde{F}_{X Y \mid W_{1}, W_{2}}\left(x, \infty \mid w_{1}, w_{2}\right)=C\left(F_{X \mid W_{1}}\left(x \mid w_{1}\right), 1 \mid w_{1}, w_{2}\right)=F_{X \mid W_{1}}\left(x \mid w_{1}\right)$, the conditional distribution of $X \mid W_{1}$, which is the conditional marginal distribution of $(X, Y) \mid W_{1}$. But $\tilde{F}_{X Y \mid W_{1}, W_{2}}\left(\infty, y \mid w_{1}, w_{2}\right)=C\left(1, F_{Y \mid W_{2}}\left(y, \mid w_{2}\right) \mid w_{1}, w_{2}\right)=$ $F_{Y \mid W_{2}}\left(y, \mid w_{2}\right)$, the conditional distribution of $Y \mid W_{2}$, which is the conditional marginal distribution of $(X, Y) \mid W_{2}$. Thus the function $\tilde{F}_{X Y \mid W_{1}, W_{2}}$ will not be the joint distribution of $(X, Y) \mid\left(W_{1}, W_{2}\right)$ in general.

The only case when $\tilde{F}_{X Y \mid W_{1}, W_{2}}$ will be the joint distribution of $(X, Y) \mid\left(W_{1}\right.$, $\left.W_{2}\right)$ is when $F_{X \mid W_{1}}\left(x \mid w_{1}\right)=F_{X \mid W_{1}, W_{2}}\left(x \mid w_{1}, w_{2}\right)$ for all $\left(x, w_{1}, w_{2}\right) \in \mathbb{R} \times \mathcal{W}_{1} \times \mathcal{W}_{2}$ and $F_{Y \mid W_{2}}\left(y, \mid w_{2}\right)=F_{Y \mid W_{1}, W_{2}}\left(y, \mid w_{1}, w_{2}\right)$ for all $\left(y, w_{1}, w_{2}\right) \in \mathbb{R} \times \mathcal{W}_{1} \times \mathcal{W}_{2}$. Although this is obviously a special case, it is not uncommon to find that certain variables affect the conditional distribution of one variable but not the other, and thus this condition is satisfied. For example, in our empirical application we find that, conditional on lags of the DM-USD exchange rate, lags of the Yen-USD exchange rate do not impact the distribution of the DM-USD exchange rate. Similarly, lags of the DM-USD exchange rate do not affect the Yen-USD exchange rate, conditional on lags of the Yen-USD exchange rate. Thus, in our case the above condition is satisfied, though it must be tested in each separate application.

The density function equivalent of (3) is useful for maximum likelihood estimation, and is easily obtained provided that $F_{X \mid Y}$ and $F_{Y \mid W}$ are differentiable and $F_{X Y \mid W}$ and $C$ are twice differentiable.

$$
\begin{aligned}
f_{X Y \mid W}(x, y \mid w) & \equiv \frac{\partial^{2} F_{X Y \mid W}(x, y \mid w)}{\partial x \partial y} \\
& =\frac{\partial F_{X \mid W}(x \mid w)}{\partial x} \cdot \frac{\partial F_{Y \mid W}(y \mid w)}{\partial y} \cdot \frac{\partial^{2} C\left(F_{X \mid W}(x \mid w), F_{Y \mid W}(y \mid w) \mid w\right)}{\partial u \partial v}
\end{aligned}
$$

$f_{X Y \mid W}(x, y \mid w) \equiv f_{X \mid W}(x \mid w) \cdot f_{Y \mid W}(y \mid w) \cdot c(u, v \mid w), \quad \forall(x, y, w) \in \overline{\mathbb{R}} \times \overline{\mathbb{R}} \times \mathcal{W}$

$$
\text { so } \quad \mathcal{L}_{X Y}=\mathcal{L}_{X}+\mathcal{L}_{Y}+\mathcal{L}_{C}
$$

where $u \equiv F_{X \mid W}(x \mid w)$, and $v \equiv F_{Y \mid W}(y \mid w), \mathcal{L}_{X Y} \equiv \log f_{X Y \mid W}(x, y \mid w), \mathcal{L}_{X} \equiv$ $\log f_{X \mid W}(x \mid w), \mathcal{L}_{Y} \equiv \log f_{Y \mid W}(y \mid w)$, and $\mathcal{L}_{C} \equiv \log c(u, v \mid w)$.

\section{THE CONDITIONAL DEPENDENCE BETWEEN THE MARK AND THE YEN}

In this section we apply the theory of conditional copulas to the modeling of the conditional bivariate distribution of the daily Deutsche mark-U.S. dollar and 
Japanese yen-U.S. dollar exchange rate returns over the period January 2, 1991, to December 31, 2001. This represents the post-unification era in Germany (East and West Germany were united in late 1989, and some financial integration was still being carried out during 1990) and includes the first 3 years of the euro's reign as the official currency of Germany. ${ }^{8}$ The Yen-USD and DM-USD (euro-USD since 1999) exchange rates are the two most heavily traded, representing close to $50 \%$ of total foreign exchange trading volume. ${ }^{9}$ Given their status, the DM-USD and Yen-USD exchange rates have been relatively widely studied; see Andersen and Bollerslev (1998), Diebold et al. (1999), Andersen et al. (2001), among others. However, there has not, to our knowledge, been any investigation of the symmetry of the dependence structure between these exchange rates.

Table 1 presents some summary statistics of the data. The data were taken from the database of Datastream International and as usual we analyze the logdifference of each exchange rate. The table shows that neither exchange rate had a significant trend over either period, both means being very small relative to the standard deviation of each series. Both series also exhibit slight negative skewness, and excess kurtosis. The Jarque-Bera test of the normality of the unconditional distribution of each exchange rate strongly rejects unconditional normality in both periods. The unconditional correlation coefficient between these two exchange rate returns indicates relatively high linear dependence prior to the introduction of the euro and weaker dependence afterward.

In specifying a model of the bivariate density of DM-USD and Yen-USD exchange rates, we must specify three models: the models for the marginal distributions of each exchange rate and the model for the conditional copula. We will first present estimation and goodness-of-fit test results for the marginal distribution models. We will then proceed to the main focus of this section: a detailed study of the results for the conditional copula models. We will examine the impact of the introduction of the euro on the joint distribution of the DM-USD and Yen-USD exchange rates by allowing the parameters of the joint distribution to change between the pre- and post-euro subsamples. Note that allowing the parameters to change pre- and post-euro is equivalent to expanding the information set to include an indicator variable that takes the value zero in the pre-euro sample and one in the post-euro sample. Recall that the same information set must be used for both margins and the copula, meaning that we must test for a structural break in the DM margin, the Yen margin, and the copula. To minimize the number of additional parameters in the models, we conducted tests for the significance of the change in each parameter, and imposed constancy on those parameters that were not significantly different in the two periods.

Maximum likelihood is the natural estimation procedure to use for our models. The procedure employed to construct the joint distribution lends itself naturally

\footnotetext{
${ }^{8}$ The mark was still used for transactions in Germany until the end of 2001, but the mark/euro exchange rate was fixed on January 1, 1999, and all international transactions were denominated in euros.

${ }^{9}$ See the Bank for International Settlements' 1996 and 2002 Central Bank Survey of Foreign Exchange and Derivatives Market Activity.
} 
TABLE 1

SUMMARY STATISTICS

\begin{tabular}{|c|c|c|}
\hline & DM-USD & Yen-USD \\
\hline & \multicolumn{2}{|c|}{ Pre-Euro } \\
\hline Mean & 0.005 & -0.009 \\
\hline Std. Dev. & 0.676 & 0.734 \\
\hline Skewness & -0.015 & -0.749 \\
\hline Kurtosis & 4.964 & 9.296 \\
\hline Jarque-Bera statistic & $327.4^{*}$ & $3560^{*}$ \\
\hline ARCH LM statistic & $165.6^{*}$ & $217.4^{*}$ \\
\hline Linear correlation & \multicolumn{2}{|c|}{0.509} \\
\hline \multirow[t]{2}{*}{ Number of obs. } & \multicolumn{2}{|c|}{2046} \\
\hline & \multicolumn{2}{|c|}{ Post-Euro } \\
\hline Mean & 0.036 & 0.019 \\
\hline Std. Dev. & 0.662 & 0.674 \\
\hline Skewness & -0.503 & -0.229 \\
\hline Kurtosis & 4.283 & 4.247 \\
\hline Jarque-Bera statistic & $84.59^{*}$ & $55.97^{*}$ \\
\hline ARCH LM statistic & 15.88 & $49.18^{*}$ \\
\hline Linear correlation & \multicolumn{2}{|c|}{0.124} \\
\hline Number of obs. & \multicolumn{2}{|c|}{773} \\
\hline
\end{tabular}

NOTE: This table presents some summary statistics of the data used in this article. The data are 100 times the log-differences of the daily Deutsche mark-U.S. dollar and Japanese yen-U.S. dollar exchange rates. The sample period runs 11 years from January 1991 to December 2001, yielding 2,819 observations in total; 2,046 prior to the introduction of the euro on January 1, 1999 and 773 after the introduction of the euro. The ARCH LM test of Engle (1982) is conducted using 10 lags. An asterisk (*) indicates a rejection of the null hypothesis at the 0.05 level.

to multistage estimation of the model, where we estimate the two marginal distribution models separately, and then estimate the copula model in a final stage, see Patton (forthcoming) for details. Although estimating all of the coefficients simultaneously yields the most efficient estimates, the large number of parameters can make numerical maximization of the likelihood function difficult. Under standard conditions, the estimates obtained are consistent and asymptotically normal.

3.1. The Models for the Marginal Distributions. The models employed for the marginal distributions are presented below. We will denote the log-difference of the DM-USD exchange rate as the variable $X_{t}$ and the log-difference of the Yen-USD exchange rate as the variable $Y_{t}$.

$$
\begin{aligned}
X_{t} & =\mu_{x}+\phi_{1 x} X_{t-1}+\varepsilon_{t} \\
\sigma_{x, t}^{2} & =\omega_{x}+\beta_{x} \sigma_{x, t-1}^{2}+\alpha_{x} \varepsilon_{t-1}^{2} \\
\sqrt{\frac{v_{x}}{\sigma_{x, t}^{2}\left(v_{x}-2\right)}} \cdot \varepsilon_{t} & \sim i i d t_{v_{x}}
\end{aligned}
$$




$$
\begin{aligned}
Y_{t} & =\mu_{y}+\phi_{1 y} Y_{t-1}+\phi_{10 y} Y_{t-10}+\eta_{t} \\
\sigma_{y, t}^{2} & =\omega_{y}+\beta_{y} \sigma_{y, t-1}^{2}+\alpha_{y} \eta_{t-1}^{2} \\
\sqrt{\frac{v_{y}}{\sigma_{y, t}^{2}\left(v_{y}-2\right)}} \cdot \eta_{t} & \sim i i d t_{v_{y}}
\end{aligned}
$$

The marginal distribution for the DM-USD exchange rate is assumed to be completely characterized by an $\operatorname{AR}(1), t$-GARCH$(1,1)$ specification, whereas the marginal distribution for the Yen-USD exchange rate is assumed to be characterized by an $\operatorname{AR}(1,10), t$-GARCH $(1,1)$ specification. ${ }^{10}$ We will call the above specifications the "copula models" for the marginal distributions, as they are to be used with the copula models introduced below.

The parameter estimates and standard errors for marginal distribution models are presented in Table 2. Table 3 shows that we only needed univariate models for these two marginal distributions: No lags of the "other" variable were significant in the conditional mean or variance specifications. This simplification will not always hold, and it should be tested in each individual case. In the DM margin, all parameters except for the degrees of freedom parameter changed significantly following the introduction of the euro. The drift term in the mean increased from 0.01 to 0.07 , reflecting the sharp depreciation in the euro in its first 3 years. In the yen margin, only the degrees of freedom changed, from 4.30 to 6.82, indicating a "thinning" of the tails of the Yen-USD exchange rate. The $t$-statistic ( $p$-value) for the significance of difference in the degrees of freedom parameters between the two exchange rates was $2.09(0.04)$ for the pre-euro period and $-0.46(0.64)$ in the post-euro period, indicating a significant difference prior to the break, but no significant difference afterwards. ${ }^{11,12}$ The significant difference in degrees of freedom parameters in the pre-euro period implies that a bivariate Student's $t$ distribution would not be a good model, as it imposes the same degrees of freedom parameter on both marginal distributions, and also on the copula.

For the purposes of comparison, we also estimate an alternative model from the existing literature (the estimation results are not presented in the interests of parsimony, but are available from the author upon request). We first model the conditional means of the two exchange rate returns series, using the models in Equations (6) and (9), and then estimate a flexible multivariate GARCH model on the residuals: the "BEKK" model introduced by Engle and Kroner (1995):

\footnotetext{
${ }^{10}$ The marginal distribution specification tests, described in the Appendix, suggested that the model for the conditional mean of the Yen-USD exchange rate return needed the 10th autoregressive lag. The 10th lag was not found to be important for the DM-USD exchange rate.

${ }^{11}$ All tests in this article will be conducted at the 5\% significance level.

12 The variance matrix used here assumed the time-varying symmetrized Joe-Clayton copula and was used to complete the joint distribution. Almost identical results were obtained when the timevarying normal copula was used.
} 
TABLE 2

RESULTS FOR THE MARGINAL DISTRIBUTIONS

\begin{tabular}{|c|c|c|}
\hline & Pre-Euro & Post-Euro \\
\hline \multicolumn{3}{|l|}{ DM-USD Margin } \\
\hline Constant & $\begin{array}{c}0.013 \\
(0.012)\end{array}$ & $\begin{array}{c}0.072 \\
(0.025)\end{array}$ \\
\hline $\operatorname{AR}(1)$ & $\begin{array}{c}0.004 \\
(0.007)\end{array}$ & $\begin{array}{c}0.025 \\
(0.034)\end{array}$ \\
\hline GARCH constant & $\begin{array}{c}0.005 \\
(0.004)\end{array}$ & $\begin{array}{c}0.000 \\
(0.004)\end{array}$ \\
\hline Lagged variance & $\begin{array}{c}0.933 \\
(0.019)\end{array}$ & $\begin{array}{c}0.994 \\
(0.043)\end{array}$ \\
\hline Lagged $e^{2}$ & $\begin{array}{c}0.059 \\
(0.016)\end{array}$ & $\begin{array}{c}0.006 \\
(0.030)\end{array}$ \\
\hline Degrees of freedom & & \\
\hline \multicolumn{3}{|l|}{ Yen-USD Margin } \\
\hline Constant & & \\
\hline $\operatorname{AR}(1)$ & & \\
\hline $\operatorname{AR}(10)$ & & \\
\hline GARCH constant & & \\
\hline Lagged variance & & \\
\hline Lagged $e^{2}$ & & \\
\hline Degrees of freedom & $\begin{array}{c}4.301 \\
(0.410)\end{array}$ & $\begin{array}{c}6.824 \\
(1.517)\end{array}$ \\
\hline
\end{tabular}

NOTE: Here we report the maximum likelihood estimates, with asymptotic standard errors in parentheses, of the parameters of the marginal distribution models for the two exchange rates. The columns refer to the period before or after the introduction of the euro on January 1, 1999. If a parameter did not change following the introduction of the euro, then it is listed in the center of these two columns.

$$
\Sigma_{t}=C C^{\prime}+B \Sigma_{t-1} B^{\prime}+A e_{t-1} e_{t-1}^{\prime} A^{\prime}
$$

where $\Sigma_{t} \equiv\left[\begin{array}{cc}\sigma_{x, t}^{2} & \sigma_{x y, t} \\ \sigma_{x y, t} & \sigma_{y, t}^{2}\end{array}\right], C \equiv\left[\begin{array}{cc}c_{11} & 0 \\ c_{12} & c_{22}\end{array}\right], B \equiv\left[\begin{array}{cc}b_{11} & b_{12} \\ b_{21} & b_{22}\end{array}\right], A \equiv\left[\begin{array}{cc}a_{11} & a_{12} \\ a_{21} & a_{22}\end{array}\right], e_{t} \equiv\left[\varepsilon_{t} \eta_{t}\right]^{\prime}, \sigma_{x, t}^{2}$ is the conditional variance of $X$ at time $t$, and $\sigma_{x y, t}$ is the conditional covariance between $X$ and $Y$ at time $t$. We use a bivariate standardized Student's $t$ distribution for the standardized residuals. We include this model as a benchmark density model obtained using techniques previously presented in the literature. When coupled with bivariate Student's $t$ innovations, the BEKK model is one of the most flexible conditional multivariate distribution models currently available, along with the multivariate regime switching model; see Ang and Bekaert 
TABLE 3

TESTING THE INFLUENCE OF THE “OTHER” VARIABLE IN THE MEAN AND VARIANCE MODELS

\begin{tabular}{lcc}
\hline & \multicolumn{2}{c}{$p$-Value } \\
\cline { 2 - 3 } & Pre-Euro & Post-Euro \\
\hline$X_{t-1}$ in conditional mean model for $Y_{t}$ & 0.72 & 0.81 \\
$Y_{t-1}$ and $Y_{t-10}$ in conditional mean model for $X_{t}$ & 0.33 & 0.38 \\
$\varepsilon_{t-1}^{2}$ in conditional variance model for $Y_{t}$ & 0.25 & 0.65 \\
$\eta_{t-1}^{2}$ and $\eta_{t-10}^{2}$ in conditional variance model for $X_{t}$ & 0.66 & 0.83 \\
\hline
\end{tabular}

NOTE: This presents the results of tests of the conditional mean and variance models presented in Equations (6), (7), (9), and (10). We report $p$-values on tests that the variables listed have coefficients equal to zero; a $p$-value greater than 0.05 means we cannot reject the null at the 0.05 level. We test whether the first lag of the DM-USD exchange rate is important for the conditional mean of the Yen-USD exchange rate by regressing the residuals $\eta_{t}$ on $X_{t-1}$ and testing that the coefficient on $X_{t-1}$ is equal to zero. Similarly, we test whether the 1 and 10 lags of the Yen-USD exchange rate are important for the conditional mean of the DM-USD exchange rate by regressing the residuals $\varepsilon_{t}$ on $Y_{t-1}$ and $Y_{t-10}$ and testing that both coefficients are equal to zero. To test the conditional variance models, we regress the standardized squared residuals of one exchange rate on the lagged squared residuals of the other exchange rate, and test that the coefficient(s) on the lagged squared residuals of the other exchange rate is (are) zero.

(2002), for example. The main cost of the BEKK models is that they quickly become unwieldy in higher dimension problems, ${ }^{13}$ and are quite difficult to estimate even for bivariate problems when the Student's $t$ distribution is assumed, as all parameters of this model must be estimated simultaneously. The parameters of this model are allowed to break following the introduction of the euro, and we also see a thinning of tails here: The estimated degrees of freedom parameter changed from 5.42 to 7.82. In both subperiods the normal distribution BEKK model was rejected (with $p$-values of less than 0.01 ) in favor of the more flexible Student's $t$ BEKK model, and so we focus solely on the Student's $t$ BEKK model.

Modeling the conditional copula requires that the models for the marginal distributions are indistinguishable from the true marginal distributions. If we use a misspecified model for the marginal distributions, then the probability integral transforms will not be Uniform $(0,1)$, and so any copula model will automatically be misspecified. Thus testing for marginal distribution model misspecification is a critical step in constructing multivariate distribution models using copulas. In the Appendix, we outline some methods for conducting such tests.

In Table 4 we present the LM tests for serial independence of the probability integral transforms, $U$ and $V$, and the Kolmogorov-Smirnov $(\mathrm{K}-\mathrm{S})$ tests of the density specification. The BEKK marginal distribution models and the copulabased marginal models pass the LM and KS tests at the 0.05 level, though the BEKK model would fail three of the KS tests at the 0.10 level. We also employ the hit tests discussed in the Appendix to check for the correctness of the specification

\footnotetext{
${ }^{13}$ Kearney and Patton (2000) estimated a five-dimension BEKK model on European exchange rates. We have not seen any applications of the BEKK model to problems of higher dimensions than this.
} 
TABLE 4

TESTS OF THE MARGINAL DISTRIBUTION MODELS

\begin{tabular}{llllll}
\hline & \multicolumn{2}{c}{ Student's $t$ BEKK } & & \multicolumn{2}{c}{ Copula Margins } \\
\cline { 2 - 3 } \cline { 5 - 6 } & DM & Yen & & DM & Yen \\
& & & Pre-Euro & & \\
First moment LM test & 0.14 & 0.43 & 0.16 & 0.73 \\
Second moment LM test & 0.49 & 0.14 & 0.54 & 0.49 \\
Third moment LM test & 0.77 & 0.16 & 0.79 & 0.48 \\
Fourth moment LM test & 0.87 & 0.26 & 0.87 & 0.55 \\
K-S test & 0.84 & 0.07 & 0.96 & 0.76 \\
Joint hit test & 0.39 & 0.14 & 0.70 & 0.08 \\
& & & Post-Euro & & \\
First moment LM test & 0.75 & 0.65 & 0.77 & 0.81 \\
Second moment LM test & 0.77 & 0.57 & 0.80 & 0.57 \\
Third moment LM test & 0.71 & 0.42 & 0.74 & 0.38 \\
Fourth moment LM test & 0.67 & 0.31 & 0.70 & 0.27 \\
K-S test & 0.09 & 0.67 & 0.15 & 0.32 \\
Joint hit test & 0.93 & 0.91 & 0.99 & 0.97 \\
& & & & \\
First moment LM test & 0.15 & 0.32 & 0.21 & 0.32 \\
Second moment LM test & 0.44 & 0.09 & 0.54 & 0.09 \\
Third moment LM test & 0.64 & 0.09 & 0.70 & 0.09 \\
Fourth moment LM test & 0.75 & 0.14 & 0.79 & 0.14 \\
K-S test & 0.45 & 0.06 & 0.14 & 0.43 \\
Joint hit test & 0.61 & 0.08 & 0.87 & 0.11 \\
\hline
\end{tabular}

NOTE: This table presents the $p$-values from LM tests of serial independence of the first four moments of the variables $U_{t}$ and $V_{t}$, described in the text, from the two models: a BEKK model for variance with Student's $t$ innovations and marginal models to use with copulas. We regress $\left(u_{t}-\bar{u}\right)^{k}$ and $\left(v_{t}-\bar{v}\right)^{k}$ on 10 lags of both variables, for $k=1,2,3,4$. The test statistic is $(T-20) \cdot R^{2}$ for each regression and is distributed under the null as $\chi_{20}^{2}$. Any $p$-value less than 0.05 indicates a rejection of the null hypothesis that the particular model is well specified. We also report the $p$-value from the Kolmogorov-Smirnov (KS) tests for the adequacy of the distribution model. Finally, we report the $p$-value from a joint test that the density model fits well in the five regions described in the body, using the "hit" test described in the Appendix.

in particular regions of the support. ${ }^{14}$ In the interests of parsimony, we present only the joint hit test results; the results for the individual regions are available on request. All models pass the joint hit test at the 0.05 level, though the BEKK model for the Yen would fail at the 0.10 level.

\footnotetext{
${ }^{14}$ We use five regions: the lower $10 \%$ tail, the interval from the 10 th to the 25 th quantile, the interval from the 25 th to the 75 th quantile, the interval from the 75 th to the 90 th quantile, and the upper $10 \%$ tail. These regions represent economically interesting subsets of the support-the upper and lower tails are notoriously difficult to fit, and so checking for correct specification there is important, whereas the middle $50 \%$ of the support contains the "average" observations. We use as regressors (" $Z_{j t}$ " using the notation in the Appendix) a constant, to check that the model implies the correct proportion of hits, and three variables that count the number of hits in that region, and the corresponding region of the other variable, in the last 1,5 , and 10 days, to check that the model dynamics are correctly specified. The $\lambda_{j}$ functions are set to simple linear functions of the parameters and the regressors: $\lambda_{j}\left(Z_{j t}, \beta_{j}\right)=$ $Z_{j t} \cdot \beta_{j}$.
} 
3.2. The Models for the Copula. Many of the copulas presented in the statistics literature are best suited to variables that take on joint extreme values in only one direction: survival times (Clayton, 1978), concentrations of particular chemicals (Cook and Johnson, 1981), or flood data (Oakes, 1989). Equity returns have been found to take on joint negative extremes more often than joint positive extremes, leading to the observation that "stocks tend to crash together but not boom together." No such empirical evidence is yet available for exchange rates, and the asymmetric central bank behavior and currency portfolio rebalancing stories given in the introduction could lead to asymmetric dependence between exchange rates in either direction. This compels us to be flexible in selecting a copula to use: It should allow for asymmetric dependence in either direction and should nest symmetric dependence as a special case. We will specify and estimate two alternative copulas, the "symmetrized Joe-Clayton" copula and the normal (or Gaussian) copula, both with and without time variation. The normal copula may be considered the benchmark copula in economics, though Chen et al. (2004) find evidence against the bivariate normal copula for many exchange rates. The reason for our interest in the symmetrized Joe-Clayton specification is that although it nests symmetry as a special case, it does not impose symmetric dependence on the variables like the normal copula.

3.2.1. The symmetrized Joe-Clayton copula. The first copula that will be used is a modification of the "BB7" copula of Joe (1997). We refer to the BB7 copula as the Joe-Clayton copula, as it is constructed by taking a particular Laplace transformation of Clayton's copula. The Joe-Clayton copula is

$$
\begin{aligned}
C_{J C}\left(u, v \mid \tau^{U}, \tau^{L}\right) & =1-\left(1-\left\{\left[1-(1-u)^{\kappa}\right]^{-\gamma}+\left[1-(1-v)^{\kappa}\right]^{-\gamma}-1\right\}^{-1 / \gamma}\right)^{1 / \kappa} \\
\text { where } \quad \kappa & =1 / \log _{2}\left(2-\tau^{U}\right) \\
\gamma & =-1 / \log _{2}\left(\tau^{L}\right) \\
14) \quad \text { and } \quad \tau^{U} & \in(0,1), \quad \tau^{L} \in(0,1)
\end{aligned}
$$

The Joe-Clayton copula has two parameters, $\tau^{U}$ and $\tau^{L}$, which are measures of dependence known as tail dependence. These measures of dependence are defined below.

Definition 2. If the limit

$$
\lim _{\varepsilon \rightarrow 0} \operatorname{Pr}[U \leq \varepsilon \mid V \leq \varepsilon]=\lim _{\varepsilon \rightarrow 0} \operatorname{Pr}[V \leq \varepsilon \mid U \leq \varepsilon]=\lim _{\varepsilon \rightarrow 0} C(\varepsilon, \varepsilon) / \varepsilon=\tau^{L}
$$

exists, then the copula $C$ exhibits lower tail dependence if $\tau^{L} \in(0,1]$ and no lower tail dependence if $\tau^{L}=0$. Similarly, if the limit

$$
\begin{aligned}
& \lim _{\delta \rightarrow 1} \operatorname{Pr}[U>\delta \mid V>\delta] \\
& \quad=\lim _{\delta \rightarrow 1} \operatorname{Pr}[V>\delta \mid U>\delta]=\lim _{\delta \rightarrow 1}(1-2 \delta+C(\delta, \delta)) /(1-\delta)=\tau^{U}
\end{aligned}
$$


exists, then the copula $C$ exhibits upper tail dependence if $\tau^{U} \in(0,1]$ and no upper tail dependence if $\tau^{U}=0$.

Tail dependence captures the behavior of the random variables during extreme events. Informally, in our application, it measures the probability that we will observe an extremely large depreciation (appreciation) of the yen against the USD, given that the DM has had an extremely large depreciation (appreciation) against the USD. Note that it does not matter which of the two currencies one conditions on the dollar having appreciated/depreciated against. The normal copula has $\tau^{U}=\tau^{L}=0$ for correlation less than one (see Embrechts et al., 2001), meaning that in the extreme tails of the distribution the variables are independent. The JoeClayton copula allows both upper and lower tail dependence to range anywhere from zero to one freely of each other.

One major drawback of the Joe-Clayton copula is that even when the two tail dependence measures are equal, there is still some (slight) asymmetry in the Joe-Clayton copula, due to simply the functional form of this copula. A more desirable model would have the tail dependence measures completely determining the presence or absence of asymmetry. To this end, we propose the "symmetrized Joe-Clayton" copula:

$$
\begin{aligned}
& C_{S J C}\left(u, v \mid \tau^{U}, \tau^{L}\right) \\
& \quad=0.5 \cdot\left(C_{J C}\left(u, v \mid \tau^{U}, \tau^{L}\right)+C_{J C}\left(1-u, 1-v \mid \tau^{L}, \tau^{U}\right)+u+v-1\right)
\end{aligned}
$$

The symmetrized Joe-Clayton (SJC) copula is clearly only a slight modification of the original Joe-Clayton copula, but by construction it is symmetric when $\tau^{U}=\tau^{L}$. From an empirical perspective, the fact that the SJC copula nests symmetry as a special case makes it a more interesting specification than the Joe-Clayton copula.

3.2.2. Parameterizing time variation in the conditional copula. There are many ways of capturing possible time variation in the conditional copula. We will assume that the functional form of the copula remains fixed over the sample whereas the parameters vary according to some evolution equation. This is in the spirit of Hansen's (1994) "autoregressive conditional density" model. An alternative to this approach may be to allow also for time variation in the functional form using a regime switching copula model, as in Rodriguez (2003), for example. We do not explore this possibility here.

The difficulty in specifying how the parameters evolve over time lies in defining the forcing variable for the evolution equation. Unless the parameter has some interpretation, as the parameters of the Gaussian and SJC copulas do, it is very difficult to know what might (or should) influence it to change. We propose the following evolution equations for the SJC copula:

$$
\tau_{t}^{U}=\Lambda\left(\omega_{U}+\beta_{U} \tau_{t-1}^{U}+\alpha_{U} \cdot \frac{1}{10} \sum_{j=1}^{10}\left|u_{t-j}-v_{t-j}\right|\right)
$$




$$
\tau_{t}^{L}=\Lambda\left(\omega_{L}+\beta_{L} \tau_{t-1}^{L}+\alpha_{L} \cdot \frac{1}{10} \sum_{j=1}^{10}\left|u_{t-j}-v_{t-j}\right|\right)
$$

where $\Lambda(x) \equiv\left(1+e^{-x}\right)^{-1}$ is the logistic transformation, used to keep $\tau^{U}$ and $\tau^{L}$ in $(0,1)$ at all times. ${ }^{15}$

In the above equations, we propose that the upper and lower tail dependence parameters each follow something akin to a restricted $\operatorname{ARMA}(1,10)$ process. The right-hand side of the model for the tail dependence evolution equation contains an autoregressive term, $\beta_{U} \tau_{t-1}^{U}$ and $\beta_{L} \tau_{t-1}^{L}$, and a forcing variable. Identifying a forcing variable for a time-varying limit probability is somewhat difficult. We propose using the mean absolute difference between $u_{t}$ and $v_{t}$ over the previous 10 observations as a forcing variable. ${ }^{16}$ The expectation of this distance measure is inversely related to the concordance ordering of copulas; under perfect positive dependence it will equal zero, under independence it equals $1 / 3$, and under perfect negative dependence it equals $1 / 2$.

The second copula considered, the normal copula, is the dependence function associated with bivariate normality, and is given by

$$
C(u, v \mid \rho)=\int_{-\infty}^{\Phi^{-1}(u)} \int_{-\infty}^{\Phi^{-1}(v)} \frac{1}{2 \pi \sqrt{\left(1-\rho^{2}\right)}} \exp \left\{\frac{-\left(r^{2}-2 \rho r s+s^{2}\right)}{2\left(1-\rho^{2}\right)}\right\} d r d s,
$$

where $\Phi^{-1}$ is the inverse of the standard normal c.d.f. We propose the following evolution equation for $\rho_{t}$ :

$$
\rho_{t}=\tilde{\Lambda}\left(\omega_{\rho}+\beta_{\rho} \cdot \rho_{t-1}+\alpha \cdot \frac{1}{10} \sum_{j=1}^{10} \Phi^{-1}\left(u_{t-j}\right) \cdot \Phi^{-1}\left(v_{t-j}\right)\right)
$$

where $\tilde{\Lambda}(x) \equiv\left(1-e^{-x}\right)\left(1+e^{-x}\right)^{-1}=\tanh (x / 2)$ is the modified logistic transformation, designed to keep $\rho_{t}$ in $(-1,1)$ at all times. Equation (19) reveals that we again assume that the copula parameter follows an $\operatorname{ARMA}(1,10)$-type process: We include $\rho_{t-1}$ as a regressor to capture any persistence in the dependence parameter, and the mean of the product of the last 10 observations of the transformed variables $\Phi^{-1}\left(u_{t-j}\right)$ and $\Phi^{-1}\left(v_{t-j}\right)$, to capture any variation in dependence. ${ }^{17}$

\footnotetext{
${ }^{15}$ We thank a referee for pointing out that using $\Lambda^{-1}\left(\tau_{t-1}^{L}\right)$ and $\Lambda^{-1}\left(\tau_{t-1}^{U}\right)$, instead of $\tau_{t-1}^{L}$ and $\tau_{t-1}^{U}$, in the evolution equations would lead to a process that is an autoregression in this transform of the tail dependence parameters. A similar comment also applies to the evolution equation for the normal copula, presented in Equation (19) below.

${ }^{16}$ A few variations on this forcing variable were tried, such as weighting the observations by how close they are to the extremes or by using an indicator variable for whether the observation was in the first, second, third, or fourth quadrant. No significant improvement was found, and so we have elected to use the simplest model.

${ }^{17}$ Averaging $\Phi^{-1}\left(u_{t-j}\right) \cdot \Phi^{-1}\left(v_{t-j}\right)$ over the previous 10 lags was done to keep the copula specification here comparable with that of the time-varying symmetrized Joe-Clayton copula.
} 
3.3. Results for the Copulas. We now present the main results of this article: the estimation results for the normal and symmetrized Joe-Clayton (SJC) models. For the purposes of comparison, we also present the results for these two copulas when no time variation in the copula parameters is assumed. It should be pointed out, though, that neither of these copulas is closed under temporal aggregation, so if the conditional copula of $\left(X_{t}, Y_{t}\right)$ is normal or $\mathrm{SJC}$, the unconditional copula will not in general be normal or SJC. The estimation results are presented in Table $5 .{ }^{18}$

All of the parameters in the time-varying normal copula were found to significantly change following the introduction of the euro, and a test of the significance of a break for this copula yielded a $p$-value of less than 0.01 . Using quadrature, ${ }^{19}$ we computed the implied time path of conditional correlation between the two exchange rates, and present the results in Figure 2. This figure shows quite clearly the structural break in dependence that occurred upon the introduction of the euro. The level and the dynamics of (linear) dependence both clearly change. The $p$-value from the test for a change in level only was less than 0.01 , and the $p$-value from a test for a change in dynamics given a change in level was also less than 0.01 , confirming this conclusion.

For the time-varying SJC copula, only the level of dependence was found to significantly change; the dynamics of conditional upper and lower tail dependence were not significantly different. The significance of the change in level was less than 0.01. For the purposes of comparing the results for the SJC copula with the normal copula, we present in Figure 3 the conditional correlation between the two exchange rates implied by the SJC copula. The plot is similar to that in Figure 2, and the change in the level of linear correlation upon the introduction of the euro is again very clear.

In Figures 4 and 5 we present plots of the conditional tail dependence implied by the time-varying SJC copula model. Figure 4 confirms that the change in linear dependence also takes place in tail dependence, with average tail dependence (defined as $\left(\tau_{t}^{U}+\tau_{t}^{L}\right) / 2$ ) dropping from 0.33 to 0.03 after the break. Figure 5 shows the degree of asymmetry in the conditional copula by plotting the difference between the upper and lower conditional tail dependence measures $\left(\tau_{t}^{U}-\tau_{t}^{L}\right)$. Under symmetry, this difference would, of course, be zero. In our model, upper (lower) tail dependence measures the dependence between the exchange rates on days when the yen and mark are both depreciating (appreciating) against the USD. Our constant SJC copula results suggest that in the pre-euro period, the

\footnotetext{
18 The parameters of the constant SJC copula were found to significantly change following the introduction of the euro, and in the post-euro period the upper tail dependence parameter went to zero. As $\tau^{U} \rightarrow 0$, the SJC copula with parameters $\left(\tau^{U}, \tau^{L}\right)$ limits to an equally weighted mixture of the Clayton copula with parameter $-\left(\log _{2}\left(\tau^{L}\right)\right)^{-1}$ and the rotated "B5" copula of Joe (1997) with parameter $\left(\log _{2}\left(2-\tau^{U}\right)\right)^{-1}$. Since zero is on the boundary of the parameter space for $\tau^{U}$ in the SJC copula, we impose $\tau^{U}=0$ and only estimate $\tau^{L}$ in the post-euro period.

${ }^{19}$ We use Gauss-Legendre quadrature, with 10 nodes for each margin, leading to a total of 100 nodes. See Judd (1998) for more on this technique. Although the normal copula is parameterized by a correlation coefficient, when the margins are nonnormal this coefficient will not equal the linear correlation between the original variables. We must use quadrature, or some other method, to extract the linear correlation coefficient.
} 
TABLE 5

RESULTS FOR THE COPULA MODELS

\begin{tabular}{|c|c|c|c|}
\hline & Pre-Euro & & Post-Euro \\
\hline \multicolumn{4}{|c|}{ Constant normal copula } \\
\hline $\bar{\rho}$ & $\begin{array}{c}0.540 \\
(0.015)\end{array}$ & & $\begin{array}{c}0.137 \\
(0.038)\end{array}$ \\
\hline Copula likelihood & & 360.34 & \\
\hline \multicolumn{4}{|c|}{ Constant SJC copula } \\
\hline $\bar{\tau}^{U}$ & $\begin{array}{c}0.359 \\
(0.025)\end{array}$ & & $\begin{array}{c}0.000 \\
-\end{array}$ \\
\hline $\bar{\tau}^{L}$ & $\begin{array}{c}0.294 \\
(0.027)\end{array}$ & & $\begin{array}{c}0.093 \\
(0.038)\end{array}$ \\
\hline Copula likelihood & & 353.42 & \\
\hline \multicolumn{4}{|c|}{ Time-varying normal copula } \\
\hline Constant & $\begin{array}{c}-0.170 \\
(0.004)\end{array}$ & & $\begin{array}{c}0.146 \\
(0.095)\end{array}$ \\
\hline$\alpha$ & $\begin{array}{c}0.056 \\
(0.014)\end{array}$ & & $\begin{array}{c}0.256 \\
(0.199)\end{array}$ \\
\hline$\beta$ & $\begin{array}{c}2.509 \\
(0.010)\end{array}$ & & $\begin{array}{c}0.724 \\
(0.613)\end{array}$ \\
\hline Copula likelihood & & 372.75 & \\
\hline \multicolumn{4}{|c|}{ Time-varying SJC copula } \\
\hline Constant $^{U}$ & $\begin{array}{c}-1.721 \\
(0.228)\end{array}$ & & $\begin{array}{c}-7.756 \\
(1.483)\end{array}$ \\
\hline$\alpha^{U}$ & & $\begin{array}{c}-1.090 \\
(0.790)\end{array}$ & \\
\hline$\beta^{U}$ & & $\begin{array}{c}3.803 \\
(0.232)\end{array}$ & \\
\hline Constant $^{L}$ & $\begin{array}{c}1.737 \\
(0.611)\end{array}$ & & $\begin{array}{c}-0.437 \\
(1.241)\end{array}$ \\
\hline$\alpha^{L}$ & & $\begin{array}{c}-6.604 \\
(3.132)\end{array}$ & \\
\hline$\beta^{L}$ & & $\begin{array}{r}-4.482 \\
(0.262)\end{array}$ & \\
\hline Copula likelihood & & 374.47 & \\
\hline
\end{tabular}

NOTE: Here we report the maximum likelihood estimates, with asymptotic standard errors in parentheses, of the parameters of the copula models. The columns refer to the period before or after the introduction of the euro on January 1, 1999. If a parameter did not change following the introduction of the euro, then it is listed in the center of these two columns. We also report the copula likelihood of the models over the entire sample. The parameter $\bar{\tau}^{U}$ in the constant SJC copula for the post-euro sample was imposed to equal zero, so no standard error is given.

limiting probability of the yen depreciating heavily against the dollar, given that the mark has depreciated heavily against the dollar, is about $22 \%$ greater than the corresponding appreciation probability, meaning that the exchange rates are more dependent during depreciations against the dollar than during appreciations. This difference is significant at the 0.05 level. Further, from Figure 5 we note that conditional upper tail dependence was greater than conditional lower tail dependence on $92 \%$ of days in the pre-euro period. As we used the same forcing 


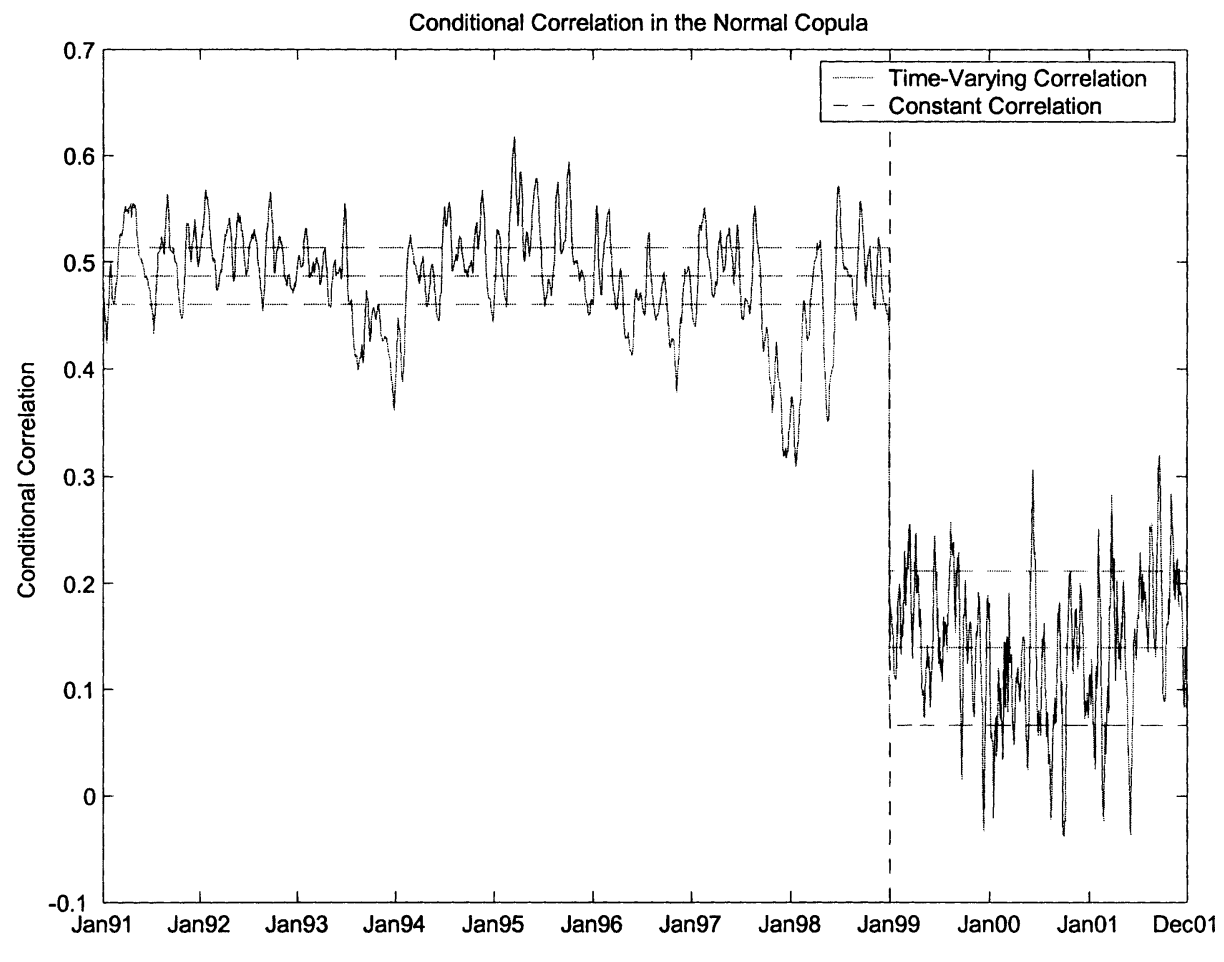

FIGURE 2

CONDITIONAL CORRELATION ESTIMATES FROM THE NORMAL COPULAS ALLOWING FOR A STRUCTURAL BREAK AT THE INTRODUCTION OF THE EURO ON JANUARY 1, 1999, WITH 95\% CONFIDENCE INTERVAL FOR THE CONSTANT CORRELATION CASE

variable in the evolution equations for both upper and lower dependence, we can formally test for the significance of asymmetry in the conditional copula by testing that the parameters of the upper tail dependence coefficient equal the parameters of the lower tail dependence coefficient. The $p$-value for this test is 0.01 in the pre-euro sample. Thus, our finding is consistent with export competitiveness preference dominating price stability preference for the Bank of Japan and/or the Bundesbank in the pre-euro period, and is also consistent with the currency portfolio rebalancing story given in the introduction. Of course, there may be other explanations for our finding.

In the post-euro period the asymmetry is reversed. The constant SJC copula results show upper tail dependence to be zero and lower tail dependence to be 0.09 , which is significantly greater than zero (the $p$-value is 0.01 ). Further, Figure 5 also shows that conditional upper tail dependence is less than conditional lower tail dependence on every day in the post-euro sample (though the $p$-value on a test of the significance of this difference is 0.16 ). These results are consistent with price stability preference dominating export competitiveness preference for the Bank of Japan and/or the European Central Bank over the post-euro sample. 


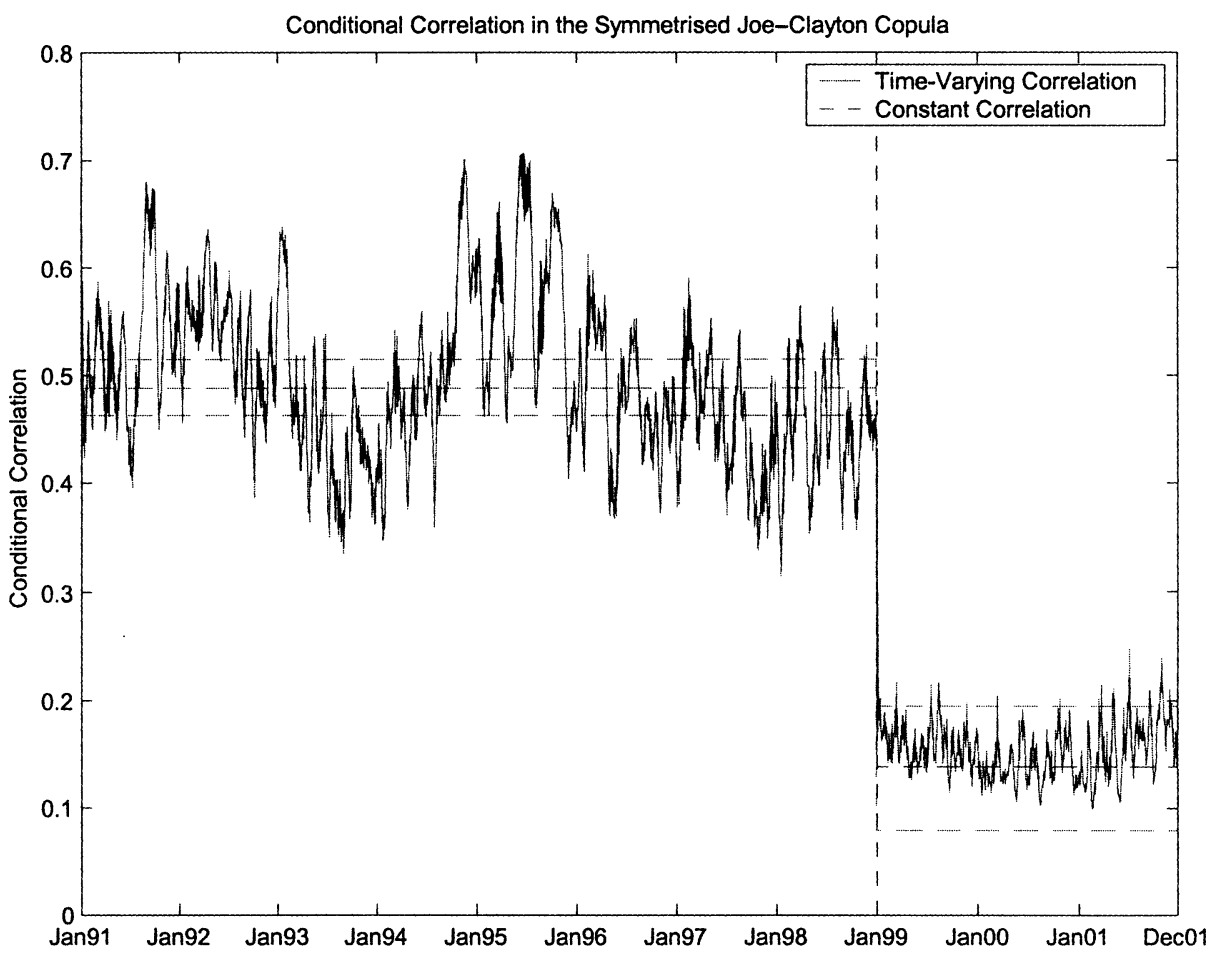

FIGURE 3

CONDITIONAL CORRELATION ESTIMATES FROM THE SYMMETRIZED JOE-CLAYTON COPULAS ALLOWING FOR A STRUCTURAL BREAK AT THE INTRODUCTION OF THE EURO ON JANUARY 1, 1999, wiTH $95 \%$ CONFIDENCE INTERVAL FOR THE CONSTANT TAIL DEPENDENCE CASE

Overall, a test that the time-varying SJC copula is symmetric over the entire sample is rejected, with $p$-value 0.02 , and the corresponding $p$-value for the constant SJC copula is also 0.02. Thus, we have strong evidence that the conditional dependence structure between the DM-dollar (euro-dollar) and yen-dollar exchange rates was asymmetric over the sample period, a finding that has not been previously reported in the empirical exchange rate literature, and one that we would not have been able to capture with standard multivariate distributions like the normal or Student's $t$. This has potentially important implications for portfolio decisions and hedging problems involving these exchange rates, as it implies that linear correlation is not sufficient to describe their dependence structure. Thus, for example, a hedge constructed using linear correlation may not offer the degree of protection it would under a multivariate normal or Student's $t$ distribution.

3.4. Goodness-of-Fit Tests and Comparisons. The evaluation of copula models is a special case of the more general problem of evaluating multivariate density models, which is discussed in the Appendix. In Table 6, we present the results of the bivariate "hit" tests. We divided the support of the copula into seven regions, each 


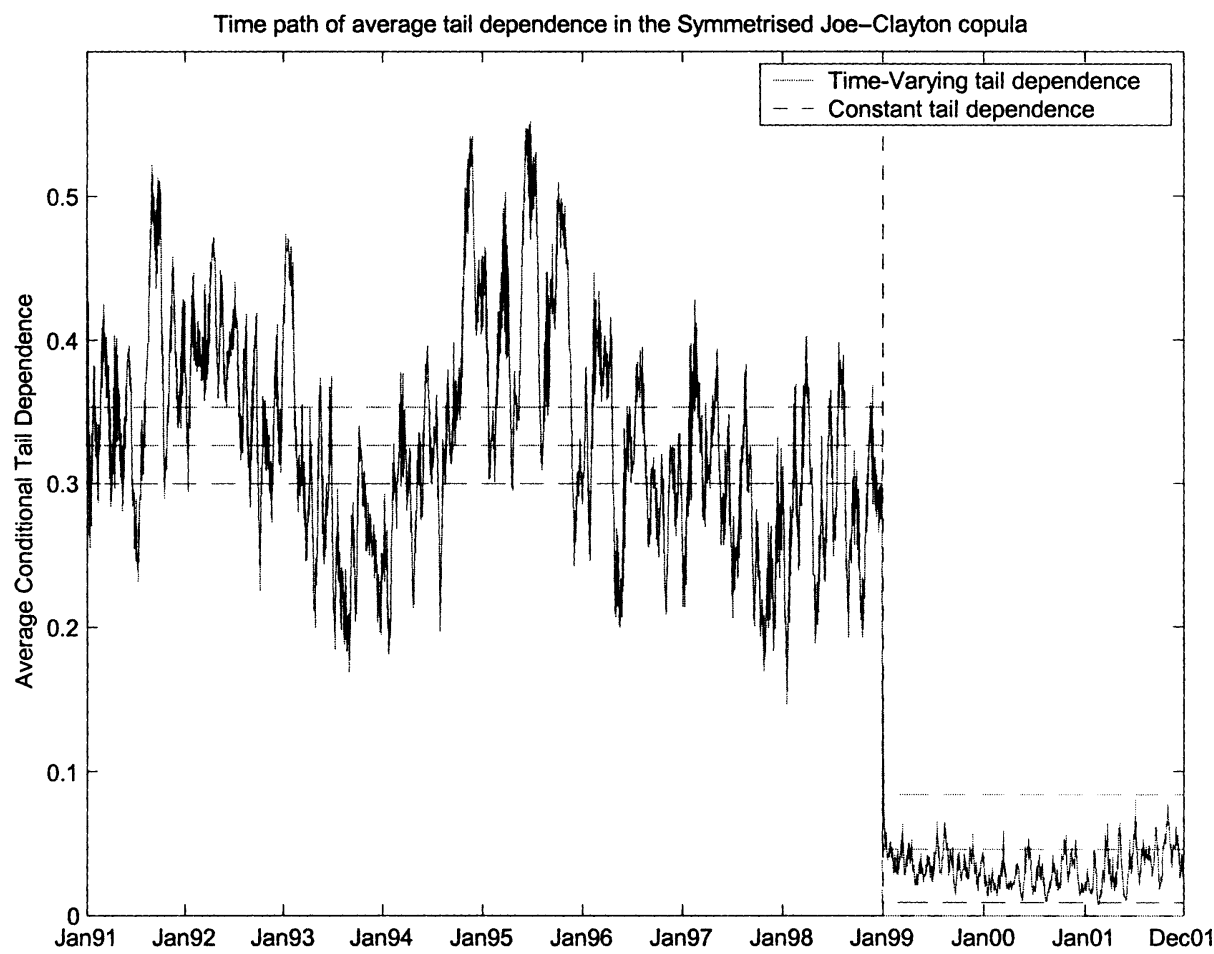

FIGURE 4

AVERAGE TAIL DEPENDENCE FROM THE SYMMETRIZED JOE-CLAYTON COPULAS ALLOWING FOR A STRUCTURAL BREAK AT THE INTRODUCTION OF THE EURO ON JANUARY 1, 1999, WITH 95\% CONFIDENCE INTERVAL FOR THE CONSTANT TAIL DEPENDENCE CASE

with an economic interpretation. ${ }^{20}$ We report only the results of the joint test that the models are well specified in all regions; the results for the individual regions are available on request. All five models pass all joint tests and all individual region tests at the 0.05 level, though the Student's $t$ BEKK model would fail two tests at the 0.10 level. Thus, although these models have quite different implications for

\footnotetext{
${ }^{20}$ Regions 1 and 2 correspond to the lower and upper joint $10 \%$ tails for each variable. The ability to correctly capture the probability of both exchange rates taking on extreme values simultaneously is of great importance to portfolio managers and macroeconomists, among others. Regions 3 and 4 represent moderately large up and down days: days in which both exchange rates were between their 10 th and 25 th, or 75 th and 90th, quantiles. Region 5 is the "median" region: days when both exchange rates were in the middle $50 \%$ of their distributions. Regions 6 and 7 are the extremely asymmetric days, those days when one exchange rate was in the upper $25 \%$ of its distribution whereas the other was in the lower $25 \%$ of its distribution. For the joint test, we define the zeroth region as that part of the support not covered by regions one to seven. We again specify a simple linear function for $\lambda_{j}$, that is: $\lambda_{j}\left(Z_{j t}, \beta_{j}\right)=Z_{j t} \cdot \beta_{j}$, and we include in $Z_{j t}$ a constant term, to capture any over- or underestimation of the unconditional probability of a hit in region $j$, and three variables that count the number of hits that occurred in the past 1,5, and 10 days, to capture any violations of the assumption that the hits are serially independent.
} 


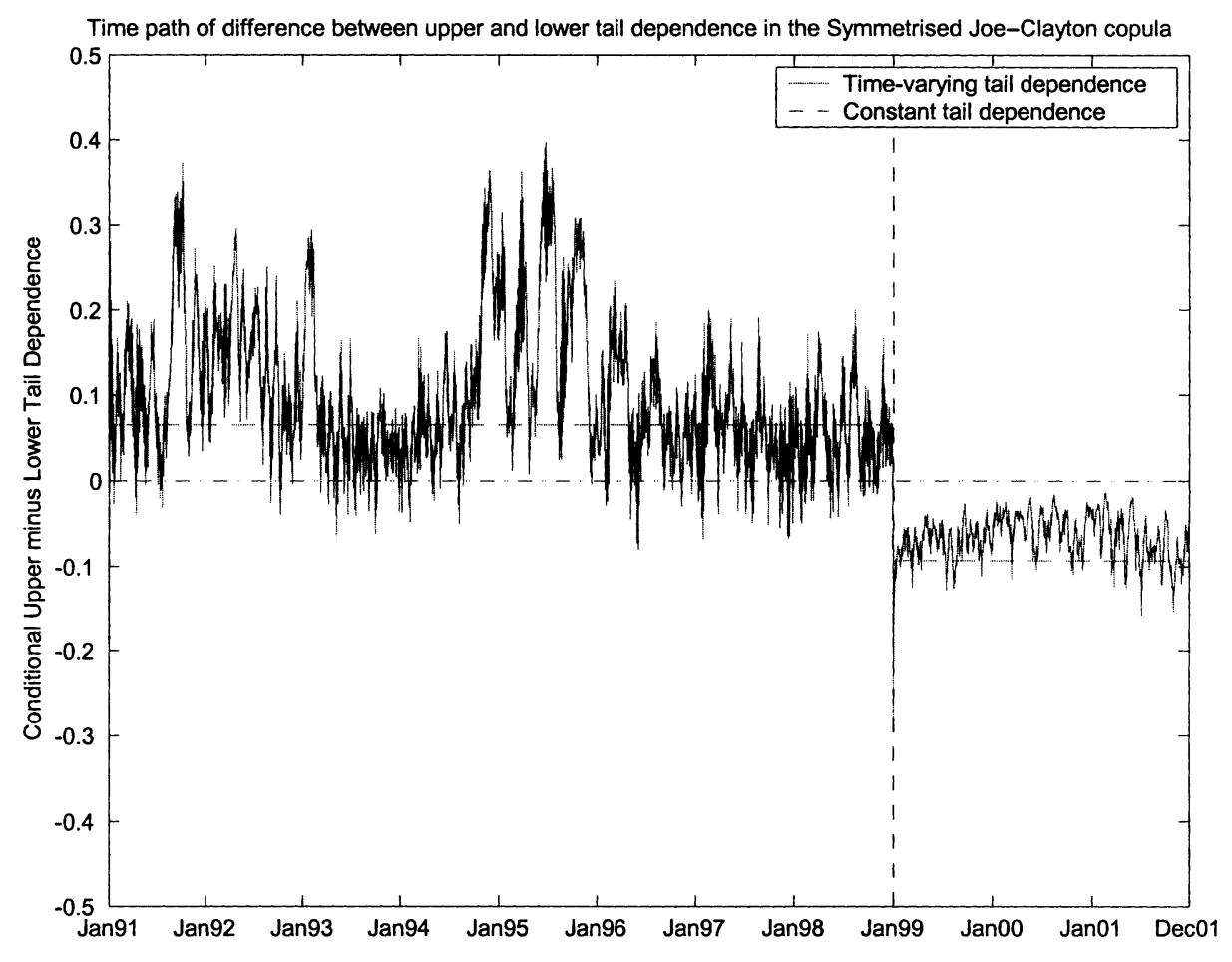

FIGURE 5

DIFFERENCE BETWEEN UPPER AND LOWER TAIL DEPENDENCE FROM THE SYMMETRIZED JOE-CLAYTON COPULAS ALLOWING FOR A STRUCTURAL BREAK AT THE INTRODUCTION OF THE EURO ON JANUARY 1, 1999

TABLE 6

JOINT HIT TEST RESULTS FOR THE COPULA MODELS

\begin{tabular}{lccccc}
\hline & $\begin{array}{c}\text { Student's } t \\
\text { BEKK }\end{array}$ & $\begin{array}{c}\text { Constant } \\
\text { Normal Copula }\end{array}$ & $\begin{array}{c}\text { Constant } \\
\text { SJC Copula }\end{array}$ & $\begin{array}{c}\text { Time-Varying } \\
\text { Normal Copula }\end{array}$ & $\begin{array}{c}\text { Time-Varying } \\
\text { SJC Copula }\end{array}$ \\
\hline Pre-euro & 0.05 & 0.46 & 0.10 & 0.62 & 0.26 \\
Post-euro & 0.93 & 0.92 & 0.97 & 0.93 & 0.97 \\
Entire sample & 0.07 & 0.65 & 0.21 & 0.74 & 0.33 \\
\hline
\end{tabular}

NOTE: We report the $p$-values from joint tests that the models are correctly specified in all regions. A $p$-value less than 0.05 indicates a rejection of the null hypothesis that the model is well specified.

asymmetric dependence and/or extreme tail dependence, the specification tests have difficulty rejecting any of them with the sample size available.

Finally, we conducted likelihood ratio tests to compare the competing models. None of the time-varying models are nested in other models, and so we used Rivers and Vuong's (2002) nonnested likelihood ratio tests. ${ }^{21}$ These tests revealed

${ }^{21}$ Rivers and Vuong (2002) show that, under some conditions, the mean of the difference in loglikelihood values for two models is asymptotically normal. When the parameters of the models are 
that none of the differences in likelihood values were significant at the 0.05 or 0.10 level. The fact that both the normal and the SJC copula models pass the goodnessof-fit tests, and are not distinguishable using the Rivers and Vuong test, indicates the difficulty these tests have in distinguishing between similar models, even with substantial amounts of data. This may be because, see Figure 1 for example, the Normal, Student's $t$, and SJC copulas are quite similar for the central region of the support; the largest differences occur in the tails, where we have less data to distinguish between the competing specifications.

\section{CONCLUSION}

In this article we investigated whether the assumption that exchange rates have a symmetric dependence structure is consistent with the data. Such an assumption is embedded in the assumption of a bivariate normal or bivariate Student's $t$ distribution. Recent work on equity returns has reported evidence that stocks tend to exhibit greater correlation during market downturns than during market upturns; see Longin and Solnik (2001) and Ang and Chen (2002) for example. Risk-averse investors with uncertainty about the state of the world can be shown to generate such a dependence structure; see Ribeiro and Veronesi (2002).

The absence of any empirical or theoretical guidance on the type of asymmetry to expect in the dependence between exchange rates compelled us to be flexible in specifying a model of the dependence structure. We discussed an extension of existing results on copulas to allow for conditioning variables, and employed it to construct flexible models of the joint density of the Deutsche mark-U.S. dollar and yen-U.S. dollar exchange rates, over the period from January 1991 to December 2001.

Standard AR- $t$ GARCH models were employed for the marginal distributions of each exchange rate, and two different copulas were estimated: the copula associated with the bivariate normal distribution and the "symmetrized Joe-Clayton" copula, which allows for general asymmetric dependence. Time variation in the dependence structure between the two exchange rates was captured by allowing the parameters of the two copulas to vary over the sample period, employing an evolution equation similar to the GARCH model for conditional variances. For comparison, we also estimated a model using the BEKK specification for the conditional covariance matrix coupled with a bivariate Student's $t$ distribution for the standardized residuals.

Asymmetric behavior of central banks in reaction to exchange rate movements is a possible cause of asymmetric dependence: A desire to maintain the competitiveness of Japanese exports to the U.S. with German exports to the U.S. would lead the Bank of Japan to intervene to ensure a matching depreciation of the yen against the dollar whenever the Deutsche mark (DM) depreciated against the

estimated via maximum likelihood, the asymptotic variance of the log-likelihood ratio is simple to compute; we do so using a Newey-West (1987) variance estimator. We use a variety of "truncation lengths" (the number of lags used to account for autocorrelation and heteroskedasticity) and found little sensitivity. 
U.S. dollar, and generate stronger dependence during depreciations of the DM and the yen against the dollar than during depreciations. Alternatively, a preference for price stability would lead the Bank of Japan to intervene to ensure a matching appreciation of the yen against the dollar whenever the DM appreciated against the U.S. dollar, and generate the opposite type of asymmetric dependence. We found evidence consistent with the scenario that export competitiveness preference dominated price stability, preference for the Bank of Japan and/or the Bundesbank in the pre-euro period, whereas price stability preference dominated export competitiveness preference for the Bank of Japan and/or the European Central Bank over the post-euro period.

Finally, we reported strong evidence of a structural break in the conditional copula following the introduction of the euro in January 1999. The level of dependence between these exchange rates fell dramatically following the break, and the conditional dependence structure went from significantly asymmetric in one direction to weakly asymmetric in the opposite direction.

\section{APPENDIX: EVALUATION OF CONDITIONAL DENSITY MODELS}

In this appendix we outline methods for conducting goodness-of-fit tests on marginal distribution and coupla models. As stated in the body of the article, the evaluation of copula models is a special case of the more general problem of evaluating multivariate density models. Diebold et al. (1998), Diebold et al. (1999), Hong (2000), Berkowitz (2001), Chen and Fan (2004), and Thompson (2002) focus on the probability integral transforms of the data in the evaluation of density models. We use the tests of Diebold et al. (1998), and employ another test, described below.

Let us denote the two transformed series as $\left\{u_{t}\right\}_{t=1}^{T}$ and $\left\{v_{t}\right\}_{t=1}^{T}$, where $u_{t} \equiv$ $F_{t}\left(x_{t} \mid W_{t-1}\right)$ and $v_{t} \equiv G_{t}\left(y_{t} \mid W_{t-1}\right)$, for $t=1,2, \ldots, T$. Diebold et al. (1998) showed that for a time series of probability integral transforms will be iid Unif $(0,1)$ if the sequence of densities is correct, and proposed testing the specification of a density model by testing whether or not the transformed series was iid, and $\operatorname{Unif}(0,1)$ in two separate stages. We follow this suggestion, and test the independence of the first four moments of $U_{t}$ and $V_{t}$, by regressing $\left(u_{t}-\bar{u}\right)^{k}$ and $\left(v_{t}-\bar{v}\right)^{k}$ on 20 lags of both $\left(u_{t}-\bar{u}\right)^{k}$ and $\left(v_{t}-\bar{v}\right)^{k}$, for $k=1,2,3,4$. We test the hypothesis that the transformed series are $\operatorname{Unif}(0,1)$ via the Kolmogorov-Smirnov test.

Our second test compares the number of observations in each bin of an empirical histogram with what would be expected under the null hypothesis. Diebold et al. (1998) suggest that such comparisons may be useful for gaining insight into where a model fails, if at all. We decompose the density model into a set of "region" models ("interval" models in the univariate case), each of which should be correctly specified under the null hypothesis that the density model is correctly specified. The specification introduced below is a simple extension of the "hit" regressions of Christoffersen (1998) and Engle and Manganelli (2004). Clements (2002) and Wallis (2003) have proposed similar extensions. We will describe our 
modification below in a general setting, and discuss the details of implementation in the body of the article.

Let $W_{t}$ be the (possibly multivariate) random variable under analysis, and denote the support of $W_{t}$ by $\mathcal{S}$. Let $\left\{R_{j}\right\}_{j=0}^{K}$ be regions in $\mathcal{S}$ such that $R_{i} \cap R_{j}=\emptyset$ if $i \neq j$, and $\cup_{j=0}^{K} R_{j}=\mathcal{S}$. Let $\pi_{j t}$ be the true probability that $W_{t} \in R_{j}$ and let $p_{j t}$ be the probability suggested by the model. ${ }^{22}$ Finally, let $\Pi_{t} \equiv\left[\pi_{0 t}, \pi_{1 t}, \ldots, \pi_{K t}\right]^{\prime}$ and $P_{t} \equiv$ $\left[p_{0 t}, p_{1 t}, \ldots, p_{K t}\right]^{\prime}$. Under the null hypothesis that the model is correctly specified, we have that $P_{t}=\Pi_{t}$ for $t=1,2, \ldots, T$. Let us define the variables to be analyzed in the tests as $H i t_{t}^{j} \equiv \mathbf{1}\left\{W_{t} \in R_{j}\right\}$, where $\mathbf{1}\{A\}$ takes the value 1 if the argument, $A$, is true and zero elsewhere, and $M_{t} \equiv \sum_{j=0}^{K} j \cdot \mathbf{1}\left\{W_{t} \in R_{j}\right\}$.

We may test that the model is adequately specified in each of the $K+1$ regions individually via tests of the hypothesis $H_{0}: \operatorname{Hit}_{t}^{j} \sim \operatorname{inid}^{23} \operatorname{Bernoulli}\left(p_{j t}\right)$ versus $H_{1}$ : $\operatorname{Hit}_{t}^{j} \sim \operatorname{Bernoulli}\left(\pi_{j t}\right)$, where $\pi_{j t}$ is a function of both $p_{j t}$, and other elements of the time $t-1$ information set thought to possibly have explanatory power for the probability of a hit. Christoffersen (1998) and Wallis (2003) modeled $\pi_{j t}$ as a first-order Markov chain, whereas Engle and Manganelli (2004) used a linear probability model. We propose using a logit model for the hits, which makes it easier to check for the influence of other variables or longer lags, and is better suited to modeling binary random variables than a linear probability model. Specifically, we propose

$$
\pi_{j t}=\pi_{j}\left(Z_{j t}, \beta_{j}, p_{j t}\right)=\Lambda\left(\lambda_{j}\left(Z_{j t}, \beta_{j}\right)-\ln \left[\frac{1-p_{j t}}{p_{j t}}\right]\right)
$$

where $\Lambda(x) \equiv\left(1+e^{-x}\right)^{-1}$ is the logistic transformation, $Z_{j t}$ is a matrix containing elements from the information set at time $t-1, \beta_{j}$ is a $\left(k_{j} \times 1\right)$ vector of parameters to be estimated, and $\lambda_{j}$ is any function of regressors and parameters such that $\lambda_{j}(Z, 0)=0$ for all $Z$. The condition on $\lambda_{j}$ is imposed so that when $\beta_{j}=0$ we have that $\pi_{j t}=\pi_{j}\left(Z_{j t}, 0, p_{j t}\right)=p_{j t}$, and thus the competing hypotheses may be expressed as $\beta_{j}=0$ versus $\beta_{j} \neq 0$. The parameter $\beta_{j}$ may be found via maximum likelihood, where the likelihood function to be maximized is $\mathcal{L}\left(\pi_{j}\left(Z_{j}, \beta_{j}, p_{j}\right) \mid H i t^{j}\right)=\Sigma_{t=1}^{T} H i t_{t}^{j} \cdot \ln \pi_{j}\left(Z_{j t}, \beta_{j}, p_{j t}\right)+(1-$ $\left.H i t_{t}^{j}\right) \cdot \ln \left(1-\pi_{j}\left(Z_{j t}, \beta_{j}, p_{j t}\right)\right)$. The test is then conducted as a likelihood ratio test, where $L R_{j} \equiv-2 \cdot\left(\mathcal{L}\left(p_{j} \mid H i t^{j}\right)-\mathcal{L}\left(\pi_{j}\left(Z_{j}, \hat{\beta}_{j}, p_{j}\right) \mid H i t^{j}\right)\right) \sim \chi_{k_{j}}^{2}$ under the null hypothesis that the model is correctly specified in region $R_{j}$.

We may test whether the proposed density model is correctly specified in all $K+1$ regions simultaneously by testing the hypothesis $H_{0}: M_{t} \sim$ inid $\operatorname{Multinomial}\left(P_{t}\right)$ versus $H_{1}: M_{t} \sim \operatorname{Multinomial}\left(\Pi_{t}\right)$, where again we specify $\Pi_{t}$ to be a function of both $P_{t}$ and variables in the time $t-1$ information set. We propose the following specification for the elements of $\Pi_{t}$ :

\footnotetext{
22 The researcher may have a particular interest in certain regions of the support (the lower tails, for example, which are important for Value-at-Risk estimation) being correctly specified. For this reason, we consider the case where the probability mass in each region is possibly unequal.

23 "inid" stands for "independent but not identically distributed."
} 


$$
\begin{aligned}
& \pi_{1}\left(Z_{t}, \boldsymbol{\beta}, P_{t}\right)=\Lambda\left(\lambda_{1}\left(Z_{1 t}, \beta_{1}\right)-\ln \left[\frac{1-p_{1 t}}{p_{1 t}}\right]\right) \\
& \pi_{j}\left(Z_{t}, \boldsymbol{\beta}, P_{t}\right)=\left(1-\sum_{i=1}^{j-1} \pi_{i t}\right) \cdot \Lambda\left(\lambda_{j}\left(Z_{j t}, \beta_{j}\right)-\ln \left[\frac{1-\sum_{i=1}^{j} p_{i t}}{p_{j t}}\right]\right), \\
& \text { for } j=2, \ldots, K
\end{aligned}
$$

$$
\pi_{0 t}=1-\sum_{j=1}^{K} \pi_{j}\left(Z_{t}, \boldsymbol{\beta}, P_{t}\right)
$$

where $Z_{t} \equiv\left[Z_{1}, \ldots, Z_{K}\right]^{\prime}$ and $\boldsymbol{\beta} \equiv\left[\beta_{1}, \ldots, \beta_{K}\right]^{\prime}$. Let the length of $\boldsymbol{\beta}$ be denoted $K_{\beta}$. This expression for $\Pi_{t}$ is specified so that $\Pi_{t}\left(Z_{t}, \mathbf{0}, P_{t}\right)=P_{t}$ for all $Z_{t}$. Further, it allows each of the elements of $\Pi_{t}$ to be a function of a set of regressors, $Z_{j t}$, while ensuring that each $\pi_{j t} \geq 0$ and that $\Sigma_{j=0}^{K} \pi_{j t}=1$. Again the competing hypotheses may be expressed as $\boldsymbol{\beta}=0$ versus $\boldsymbol{\beta} \neq 0$. The likelihood function to be maximized to obtain the parameter $\boldsymbol{\beta}$ is $\mathcal{L}(\Pi(Z, \boldsymbol{\beta}, P) \mid H i t)=\Sigma_{t=1}^{T} \Sigma_{j=0}^{K} \ln \pi_{j t} \cdot \mathbf{1}\left\{M_{t}=j\right\}$. The joint test may also be conducted as a likelihood ratio test: $L R_{A L L} \equiv-2$. $(\mathcal{L}(P \mid H i t)-\mathcal{L}(\Pi(Z, \hat{\boldsymbol{\beta}}, P) \mid H i t)) \sim \chi_{K_{\beta}}^{2}$ under the null hypothesis that the model is correctly specified in all $K$ regions.

\section{REFERENCES}

Andersen, T. G., And T. Bollerslev, "Answering the Skeptics: Yes, Standard Volatility Models Do Provide Accurate Forecasts," International Economic Review 39 (1998), 885-905.

F. X. Diebold, and P. Labys, "The Distribution of Realized Exchange Rate Volatility," Journal of the American Statistical Association 96 (2001), 42-55.

ANG, A., AND G. BeKAerT, "International Asset Allocation with Regime Shifts," Review of Financial Studies 15 (2002), 1137-87.

- AND J. Chen, "Asymmetric Correlations of Equity Portfolios," Journal of Financial Economics 63 (2002), 443-94.

Berkowitz, J., "Testing Density Forecasts, with Applications to Risk Management," Journal of Business and Economic Statistics 19 (2001), 465-74.

_- "A Conditional Heteroskedastic Time Series Model for Speculative Prices and Rates of Return," Review of Economics and Statistics 69 (1987), 542-47.

Bouyé, E., N. Gaussel, and M. Salmon, "Investigating Dynamic Dependence Using Copulae," Working Paper, City University Business School, London, 2000a.

_ - V. Durrleman, A. Nikeghbali, G. Riboulet, and T. Roncalli, "Copulas for Finance: A Reading Guide and Some Applications," Working Paper, Groupe de Recherche Opérationnelle, Crédit Lyonnais. France, 2000b.

Chen, X., And Y. Fan, "Estimation of Copula-Based Semiparametric Time Series Models," Journal of Econometrics 130 (2006), 307-35.

— AND — - "Evaluating Density Forecast via the Copula Approach," Finance Research Letters 1 (2004), 74-84.

,$--\ldots$, and A. J. Patton, "Simple Tests for Models of Dependence Between Multiple Financial Time Series, with Applications to U.S. Equity Returns and Exchange Rates," Discussion Paper 483, Financial Markets Group, London School of Economics, 2004. 
Cherubini, U., and E. Luciano, "Value at Risk Trade-off and Capital Allocation with Copulas," Economic Notes 30 (2001), 235-56.

— AND _ _ "Bivariate Option Pricing with Copulas," Applied Mathematical Finance 8 (2002), 69-85.

,,-- AND W. Vecchiato, Copula Methods in Finance (England: John Wiley \& Sons, 2004).

Christoffersen, P. F., "Evaluating Interval Forecasts," International Economic Review 39 (1998), 841-62.

Clayton, D. G., "A Model for Association in Bivariate Life Tables and Its Application in Epidemiological Studies of Familial Tendency in Chronic Disease Incidence," Biometrika 65 (1978), 141-51.

Clements, M. P., "An Evaluation of the Survey of Professional Forecasters Probability Distributions of Expected Inflation and Output Growth," Working Paper, Department of Economics, University of Warwick, 2002.

CoOK, R. D., AND M. E. Johnson, “A Family of Distributions for Modelling Non-elliptically Symmetric Multivariate Data," Journal of the Royal Statistical Society 43 (1981), 21018.

Costinot, A., T. Roncali, and J. Teiletche, "Revisiting the Dependence between Financial Markets with Copulas," Working Paper, Credit Lyonnais (2000).

Deheuvels, P., "La fonction de dépendence empirique et ses propriétés. Un test non paramétrique d'indépendence," Académie Royale de Belgique Bulletin de la Classe des Sciences 65 (1978), 274-92.

Diebold, F. X., T. Gunther, And A. S. TAy, "Evaluating Density Forecasts, with Applications to Financial Risk Management," International Economic Review 39 (1998), 863-83.

— J. HAHN, AND A. S. TAY, "Multivariate Density Forecast Evaluation and Calibration in Financial Risk Management: High-Frequency Returns on Foreign Exchange," The Review of Economics and Statistics 81 (1999), 661-73.

Embrechts, P., A. McNeil, and D. Straumann, "Correlation and Dependence Properties in Risk Management: Properties and Pitfalls," in M. Dempster, ed., Risk Management: Value at Risk and Beyond (Cambridge: Cambridge University Press, 2001), 176-223.

Engle, R. F., "Autoregressive Conditional Heteroscedasticity with Estimates of the Variance of UK Inflation," Econometrica 50 (1982), 987-1007.

— , AND K. F. KRONER, "Multivariate Simultaneous Generalized ARCH," Econometric Theory 11 (1995), 122-50.

sion Quantiles," Journal of Business and Economic Statistics 22 (2004), 367-81.

Erb, C. B., C. R. Harvey, and T. E. Viskanta, "Forecasting International Equity Correlations," Financial Analysts Journal 50 (1994), 32-45.

Fermanian, J.-D., and O. Scaillet, "Nonparametric Estimation of Copulas for Time Series," Journal of Risk 5 (2003), 25-54.

Fisher, R. A., Statistical Methods for Research Workers (Edinburgh: Oliver and Boyd, 1932).

Frees, E. W., J. Carriere, and E. A. Valdez, "Annuity Valuation with Dependent Mortality," Journal of Risk and Insurance 63 (1996), 229-61.

Frey, R., AND A. J. McNeIL, "Modelling Dependent Defaults," ETH E-Collection, 2001. Available at http://e-collection.ethbib.ethz.ch/show?type=bericht\&nr=273.

Galambos, J., The Asymptotic Theory of Extreme Order Statistics (New York: John Wiley $\&$ Sons, 1978).

Granger, C. W. J., T. Teräsvirta, and A. J. Patton, "Common Factors in Conditional Distributions for Bivariate Time Series," Journal of Econometrics (forthcoming).

Hansen, B. E., "Autoregressive Conditional Density Estimation," International Economic Review 35 (1994), 705-30.

Hartmann, P., S. Straetmans, and C. G. De Vries, "Asset Market Linkages in Crisis Periods," Review of Economics and Statistics 81 (2004), 313-26. 
Hong, Y., "Evaluation of Out-of-Sample Density Forecasts with Applications to S\&P 500 Stock Prices," Working Paper, Department of Economics, Cornell University, 2000.

Hull, J., And A. White, "Value at Risk When Daily Changes in Market Variables Are Not Normally Distributed," Journal of Derivatives 5 (1998), 9-19.

Hutchinson, T. P., And C. D. LAI, Continuous Bivariate Distributions, Emphasising Applications (Sydney: Rumsby, 1990).

Joe, H., Multivariate Models and Dependence Concepts (London: Chapman \& Hall, 1997).

JudD, K. L., Numerical Methods in Economics (Cambridge, MA: MIT Press, 1998).

KeARney, C., AND A. J. PATton, "Multivariate GARCH Modeling of Exchange Rate Volatility Transmission in the European Monetary System," The Financial Review 41 (2000), $29-48$.

Kimeldorf, G., AND A. R. SAMPSON, "Uniform Representations of Bivariate Distributions," Communications in Statistics 4 (1975), 617-27.

LI, D. X., "On Default Correlation: A Copula Function Approach," Journal of Fixed Income $9(2000), 43-54$.

LONGIN, F., AND B. SOLNIK, "Extreme Correlation of International Equity Markets," Journal of Finance 56 (2001), 649-76.

Mills, F. C., The Behaviour of Prices (New York: National Bureau of Economic Research, 1927).

Nelsen, R. B., An Introduction to Copulas (New York: Springer-Verlag, 1999).

Newey, W. K., and K. D. West, "A Simple, Positive Semidefinite, Heteroskedasticity and Autocorrelation Consistent Covariance Matrix," Econometrica 55 (1987), 703-08.

OAKes, D., "Bivariate Survival Models Induced by Frailties," Journal of the American Statistical Association 84 (1989), 487-93.

Patton, A. J., "Applications of Copula Theory in Financial Econometrics," Unpublished Ph.D. dissertation, University of California, San Diego, 2002.

, "On the Out-of-Sample Importance of Skewness and Asymmetric Dependence for Asset Allocation," Journal of Financial Econometrics 2 (2004), 130-68.

, "Estimation of Multivariate Models for Time Series of Possibly Different Lengths," Journal of Applied Econometrics, (forthcoming).

Pearson, K., "On a Method of Determining Whether a Sample of Size $n$ Supposed to Have Been Drawn from a Parent Population Having a Known Probability Integral Has Probably Been Drawn at Random,” Biometrika 25 (1933), 379-410.

Ribeiro, R., AND P. Veronesi, “The Excess Comovement of Internatonal Stock Returns in Bad Times: A Rational Expectations Equilibrium Model," Working Paper, Graduate School of Business, University of Chicago, 2002.

Rivers, D., And Q. Vuong, "Model Selection Tests for Nonlinear Dynamic Models," The Econometrics Journal 5 (2002), 1-39.

Rockinger, M., And E. Jondeau, "Conditional Dependency of Financial Series: An Application of Copulas," Working Paper, Department of Finance, HEC School of Management, France, 2001.

Rodriguez, J. C., "Measuring Financial Contagion: A Copula Approach," EURANDOM Working Paper, 2003.

Rosenberg, J. V., "Nonparametric Pricing of Multivariate Contingent Claims," Journal of Derivatives 10 (2003), 9-26.

— , And T. Schuermann, "Integrated Risk Management Using Copulas," Working Paper, Federal Reserve Bank of New York, 2004.

Rosenblatt, M., "Remarks on a Multivariate Transformation," The Annals of Mathematical Statistics 23 (1952), 470-72.

Sancetta, A., And S. Satchell, "Bernstein Approximations to the Copula Function and Portfolio Optimization," Working Paper 2001-5, Department of Applied Economics, University of Cambridge, 2001.

Sklar, A., "Fonctions de répartition à $n$ dimensions et leurs marges," Publications de l' Institut Statistique de l'Université de Paris 8 (1959), 229-31. 
Sмiтh, M. D., "Modelling Sample Selection Using Archimedean Copulas," Econometrics Journal 6 (2003), 99-123.

TAKAGI, S., "The Yen and Its East Asian Neighbors, 1980-95: Cooperation or Competition?" in T. Ito and A. O. Krueger, eds., Changes in Exchange Rates in Rapidly Developing Countries (Chicago: The University of Chicago Press, 1999), 185-207.

Thompson, S. B., "Evaluating the Goodness of Fit of Conditional Distributions, with an Application to Affine Term Structure Models," Working Paper, Department of Economics, Harvard University, 2002.

Wallis, K. F., "Chi-squared Tests of Interval and Density Forecasts, and the Bank of England's Fan Charts," International Journal of Forecasting 19 (2003), 165-75. 